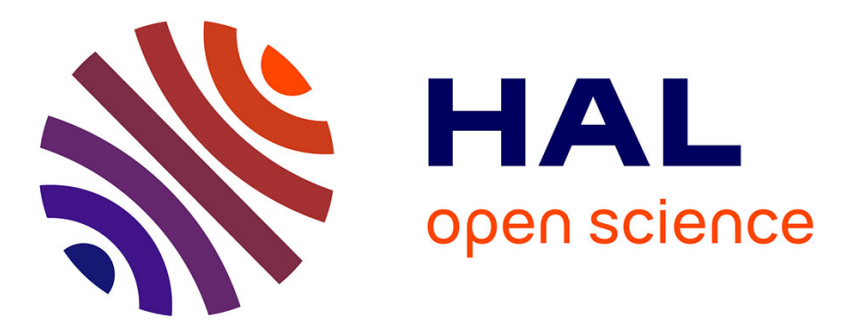

\title{
Stabilization of periodic orbits of discrete-time dynamical systems using the Prediction-Based Control: New control law and practical aspects
} Thiago P Chagas, Pierre-Alexandre Bliman, Karl H. Kienitz

\section{- To cite this version:}

Thiago P Chagas, Pierre-Alexandre Bliman, Karl H. Kienitz. Stabilization of periodic orbits of discrete-time dynamical systems using the Prediction-Based Control: New control law and practical aspects. Journal of The Franklin Institute, 2018, 355 (12), pp.4771-4793. 10.1016/j.jfranklin.2018.04.040 . hal-01941693

\section{HAL Id: hal-01941693 \\ https://hal.inria.fr/hal-01941693}

Submitted on 3 Dec 2018

HAL is a multi-disciplinary open access archive for the deposit and dissemination of scientific research documents, whether they are published or not. The documents may come from teaching and research institutions in France or abroad, or from public or private research centers.
L'archive ouverte pluridisciplinaire HAL, est destinée au dépôt et à la diffusion de documents scientifiques de niveau recherche, publiés ou non, émanant des établissements d'enseignement et de recherche français ou étrangers, des laboratoires publics ou privés. 


\title{
Preliminary version
}

Final version published in

Journal of the Franklin Institute 355 (2018) 4771-4793

\section{STABILIZATION OF PERIODIC ORBITS OF DISCRETE-TIME DYNAMICAL SYSTEMS USING THE PREDICTION-BASED CONTROL: NEW CONTROL LAW AND PRACTICAL ASPECTS.}

\author{
THIAGO P. CHAGAS \\ Dpt. de Ciências Exatas e Tecnológicas, Universidade Estadual de Santa Cruz, Rod. Jorge Amado, km 16 \\ Ilhéus, BA 45662-900, Brazil \\ tpchagas@uesc.br \\ PIERRE-ALEXANDRE BLIMAN \\ INRIA, Domaine de Voluceau \\ Rocquencourt, 78153 Le Chesnay cedex, France \\ pierre-alexandre.bliman@inria.fr \\ KARL H. KIENITZ \\ Dpt. de Sistemas e Controle, Instituto Tecnológico de Aeronáutica, Pça. Mal. Eduardo Gomes, 50 \\ São José dos Campos, SP 12228-900, Brazil \\ kienitz@ieee.org \\ Received (to be inserted by publisher)
}

\begin{abstract}
This paper tackles the stabilization of periodic orbits of nonlinear discrete-time dynamical systems with chaotic sets. The problem is approximated locally to the stabilization of linear timeperiodic systems and the theory of modern control is applied to the Prediction-Based Control, resulting in a new control law. Using numerical simulations, this control law was analyzed and compared with an optimal Delayed Feedback Control evidencing its advantages in theoretical and practical aspects.
\end{abstract}

Keywords: Prediction-Based Control; Delayed Feedback Control; Periodic Ordbits, Floquet Stability, Discrete-Time Systems.

\section{Introduction}

Stabilization of periodic orbits consists in changing the stability of an existing unstable periodic solution of a dynamical system. The stabilizing methods take advantage of the existing unstable solution to obtain a stable periodic solution using low feedback control effort. The methods can be applied to oscillatory systems where one of the performance requirements is a periodic oscillation.

Persistent oscillations are observed in many engineering problems, for example, attitude control in aerospace engineering [Mesquita et al., 2008], flutter in aeronautical engineering [Fung, 2002], shimmy in automotive and aeronautics engineering [Zhuravlev \& Klimov, 2010], power electronics in electrical engineering [Turci et al., 2009] and physiological oscillations (heart-beating, for example [Schmitt \& Ivanov, 
2007]) in biomedical engineering. Oscillations are also observed in other areas, for example, ecology [Fussmann et al., 2000] and economy [Chian et al., 2006].

Persistent oscillations are characteristic of non-linear systems with chaotic sets in their state space. In fact, if there is a chaotic set in the $n$-dimensional state space, it is known that the chaotic set is composed by an infinite number of unstable periodic orbits (UPO) and the number of UPOs per discrete period $p$ increases exponentially with $p$ [Cvitanović, 1988; Franceschini et al., 1993].

Ott et al. [1990] proposed to stabilize the UPOs embedded in the chaotic sets, namely chaos control, by small time-dependent parametric perturbations. Chaos control aims at eliminating steady state chaotic behaviour with low control effort resulting in a stable periodic oscillation. For practical applications, low control effort means reduction in actuators power, resulting in lower financial costs and increasing the equipment life-time. Although, the required parametric perturbations reduces the set of systems that the method can be applied.

Pyragas [1992] introduced the Delayed Feedback Control (DFC), a chaos control method based on statefeedback that presents the advantages of the previous one without the required parametric perturbations. Although, the DFC has also some limitations proofed for discrete-time systems, the odd-number limitation [Ushio, 1996; Yamamoto et al., 2001, 2002; Zhu \& Tian, 2005] and the impossibility to control orbits with longer periods [Zhu \& Tian, 2005], i.e., with larger Floquet exponent or equivalent, larger eigenvalue in the expanding (unstable) direction [Franceschini et al., 1993].

The Prediction-Based Control (PBC) was proposed by Ushio \& Yamamoto [1999] as an alternative to overcome the DFC limitations, maintaining its advantages. It uses the value of the state one period ahead, computed along the trajectories of the free system response as reference for the control signal.

We propose some contributions to the PBC method based on the link between chaos control and modern control theories applied and developed by control systems engineering [Sanjuán \& Grebogi, 2010; Sontag, 1998; Bittanti \& Colaneri, 2009]. For that we basically consider that the stabilization of a periodic orbit of a non-linear chaotic system can be recast as the stabilization of a linear time-periodic system. This linear system is obtained linearising the trajectories in the close vicinity of the periodic orbit and using Floquet stability theory for local orbit stability analysis. The stabilized orbits shown in this manuscript are all associated to chaotic sets, but the stabilizing methods developed can be applied to any existing UPO of an autonomous/non-autonomous discrete-time nonlinear system.

The following practical and theoretical aspects are of special concern in this contribution:

- Control systems performance specifications include transient and steady state behavior. In general, chaos control methods use the ergodicity of chaotic attractors to achieve stabilization of periodic orbits with low control effort and the drawback of large transient time [Ott et al., 1990]. We present a new PBC law based on improved sufficient stability conditions using Floquet theory [Chagas et al., 2010b,a] (see Section 2.1). This control law applied to the PBC results a control method similar to dead-beat control developed for linear discrete-time systems [Grasselli \& Lampariello, 1981]. Dead-beat main characteristic is the fast transient time, allowing the PBC strategy to accommodate transient specifications, possibly increasing control effort (see Sections 2.2 and 3);

- The new PBC law proposed does not depend on previous knowledge about the UPO position, avoiding the common problem of finding the target UPO before analytically designing of the control parameters for application. It also does not require the experimental process, suggested for the DFC [Pyragas, 1992], for tunning the control parameter. In fact, it can also be used for finding UPOs, similar to the Newton-Raphson method [Parker \& Chua, 1989] (see Sections 2.2, 3.1.1 and 3.2.1);

- Chaos control methods based on state feedback, as PBC and DFC, generally consider that all the state variables are available and the system is fully actuated [Sontag, 1998]. In practice some state variables may not be directly accessible for measurement and/or the system may be under actuated. Here we propose a control law that deals with a class of systems that are not fully actuated (see Sections 2.3 and $3.4)$;

- The PBC uses as reference the predicted state of the free system response for one target orbit period ahead. The main drawback of the PBC is the necessity of the prediction model for application and also the dependence on its precision. Methods to deal with these problems can be found in the literature of 
Model Predictive Control and Robust Control. Here we present a brief numerical robustness analysis for the new PBC law for the case of parametric uncertainty in the prediction model (see Section 3.3).

The new PBC law proposed was compared using numerical experiments with an optimal DFC applied to discrete-time systems [Chagas et al., 2012; Huijberts et al., 2009].

\subsection{Problem statement}

Consider the following discrete-time dynamical system:

$$
x_{k+1}=f\left(k, x_{k}, u_{k}\right), \quad x_{0} \text { given }
$$

where $x: \mathbb{N} \rightarrow \mathbb{R}^{n}, u: \mathbb{N} \rightarrow \mathbb{R}^{m}, k, m, n \in \mathbb{N}$ and $f: \mathbb{N} \times \mathbb{R}^{n} \times \mathbb{R}^{m} \rightarrow \mathbb{R}^{n}$ is a $p$-periodic function with respect to time $k$, i.e., by definition

$$
\forall k \in \mathbb{N}, \quad \forall x \in \mathbb{R}^{n}, \quad \forall u \in \mathbb{R}^{m}, \quad f(k+p, x, u)=f(k, x, u) .
$$

We assume moreover the existence of a $p$-periodic solution $x_{k}^{*}$ to the free system (1), i.e., the system obtained for $u \equiv 0_{m}$. In other words

$$
\forall k \in \mathbb{N}, \quad x_{k+p}^{*}=x_{k}^{*}
$$

and

$$
\forall k \in \mathbb{N}, \quad x_{k+1}^{*}=f\left(k, x_{k}^{*}, 0_{m}\right) .
$$

We assume that this periodic solution is unstable. Our ultimate objective is to synthesize periodic feedback laws $u_{k}\left(x_{k}\right)$ that stabilize it, i.e., such that

$$
\forall k \in \mathbb{N}, \quad \forall x \in \mathbb{R}^{n}, \quad u_{k+p}(x)=u_{k}(x)
$$

and such that $x^{*}$ is a stable solution of the closed-loop system

$$
x_{k+1}=f\left(k, x_{k}, u_{k}\left(x_{k}\right)\right)
$$

with $u: \mathbb{N} \times \mathbb{R}^{n} \rightarrow \mathbb{R}^{m}$ designed latter. Notice that, when the open-loop system (1) and the feedback are periodic with respect to time $k$, the same is true for the closed-loop system (5).

The control signal $u$ used in this work has to satisfy,

$$
u_{k}\left(x_{k}^{*}\right)=0, \quad k \geq 0 .
$$

That is, on the periodic orbit the control effort is zero and the unstable periodic solution $x_{k}^{*}$ of $f\left(k, x_{k}, 0\right)$ is a stable periodic solution of $f\left(k, x_{k}, u_{k}\left(x_{k}\right)\right)$.

\section{Prediction based control (PBC)}

This method was proposed by Ushio \& Yamamoto [1999] as an alternative to the DFC due to its odd number limitation[Ushio, 1996; Yamamoto et al., 2001, 2002; Zhu \& Tian, 2005]. It uses a control signal defined by

$$
u_{k}\left(x_{k}\right)=K_{k}\left(x_{k}\right)\left(\varphi\left(k+p, k, x_{k}, 0\right)-x_{k}\right),
$$

where $\varphi\left(k_{1}, k_{0}, x, 0\right)$ is the value at time $k_{1}$ of the state of (5) with $x_{k_{0}}=x$ and $u_{k}=0, k_{0} \leq k \leq k_{1}$. In other words, $\varphi\left(k_{1}, k_{0}, x, 0\right)$ is the value at time $k_{1}$ of the state along the trajectory departing from $x$ at time $k_{0}$ of the free system $\left(u_{k} \equiv 0\right)$.

A constant control gain $K$, designed using $x_{k}^{*}$, for the PBC is used in [Ushio \& Yamamoto, 1999; Morgül, 2009; Boukabou \& Mansouri, 2007]. A time varying control gain defined for the target UPO, $K_{k}\left(x_{k}^{*}\right)$, was proposed in [Hino et al., 2002]. The PBC applied via pulse-based control is presented in [Liz \& Pötzsche, 2014]. 


\subsection{Stabilization of periodic orbits by the $P B C$}

The stabilization of a periodic orbit of a non-linear system can be recast as the stabilization of a linear time-periodic system. This linear system is obtained linearising the trajectories in the close vicinity of the periodic orbit and its stability can be analyzed using the Floquet stability theory. Thus, the local stability of the periodic orbit of the non-linear system is defined by the stability of the associated linear system.

Consider the non-linear discrete-time dynamical system described by (5) and the existence of the hyperbolic periodic orbit $x^{*}$ of period $p \in \mathbb{N}$. The goal here is stabilizing the UPO $x^{*}$ of (1) subjected to the control restriction (6). The transition from $x_{k}$ to $x_{k+p}$ of (5) is defined by the closed-loop state transition map.

$$
x_{k+p}=\varphi\left(k+p, k, x_{k}, u_{k}\left(x_{k}\right)\right) .
$$

On the periodic orbit, condition (6) guarantees that any $p$-periodic orbit that satisfies (2) is also a $p$-periodic orbit of (8). Then,

$$
x_{k+p}^{*}=x_{k}^{*}=\varphi\left(k+p, k, x_{k}^{*}, u_{k}\left(x_{k}^{*}\right)\right)=\varphi\left(k+p, k, x_{k}^{*}, 0\right) .
$$

The matrix

$$
\Psi_{k}=\left.\nabla_{x} \varphi\left(k+p, k, x, u_{k}(x)\right)\right|_{x=x_{k}^{*}}
$$

is the monodromy matrix associated to the orbit $x^{*}$ of the closed-loop discrete-time system (5). The stability of the periodic orbit of (5) is related to the spectrum of the monodromy matrix, being stable if all the eigenvalues (Floquet characteristic multipliers [Bittanti \& Colaneri, 2009]) have modulus less than or equal to 1.

According to (5) and (7), the closed-loop discrete-time dynamical system controlled using the PBC is defined by

$$
x_{k+1}=\varphi\left(k+1, k, x_{k}, u_{k}\left(x_{k}\right)\right)=f\left(k, x_{k}, K_{k}\left(x_{k}\right)\left(\varphi\left(k+p, k, x_{k}, 0\right)-x_{k}\right)\right) .
$$

In the sequel, for any $x \in \mathbb{R}^{n}$ and $K \in \mathbb{R}^{q \times n}, q \in \mathbb{N}$, we use the notation

$$
\psi(k, x, K) \doteq f\left(k, x, u_{k}(x)\right), \quad u_{k}(x)=K(\varphi(k+p, k, x, 0)-x) .
$$

The first step for obtaining a sufficient stability condition for periodic orbits is to calculate the monodromy matrix $\Psi_{k}$. This task, for the closed-loop system, is done according to the proposed Lemma 1.

Lemma 1. For any p-periodic point $x_{k}^{*}, k, p \in \mathbb{N}$, of the trajectory $x^{*}$ of the closed-loop system (10), one has

$$
\Psi_{k}=\left.\prod_{l=0}^{p-1} \nabla_{x} \psi\left(k+l, x, K_{k+l}\left(x_{k+l}^{*}\right)\right)\right|_{x=x_{k+l}^{*}}
$$

and the matrices in the product are ordered from right to left for increasing indices $l$.

Proof. See Appendix A.

The interest of formula (12) is that no derivative of $K_{k}\left(x_{k}\right)$ with respect to $x_{k}$ appears in the right-hand side. Thus, Lemma 1 provides a simplification in the computation of the monodromy matrix spectrum: as indicated by (12), the dependence of the gain with respect to the state does not modify the Jacobian $\nabla_{x} \psi\left(k, x, K_{k}(x)\right)$ in the points of the periodic orbit.

The simplification provided by the Lemma 1 is used in the Theorem 1 to define a sufficient stability condition for a periodic orbit of (10).

Theorem 1. Assume the Jacobian $\left.\nabla_{x} \psi\left(k, x, K_{k}\left(x_{k}^{*}\right)\right)\right|_{x=x_{k}^{*}}$ of the system (10) is zero at least for one point of the periodic orbit $x_{k}^{*}$. Then, the periodic orbit $x^{*}$ is locally exponentially stable. 
Proof. See Appendix A.

Theorem 1 reduces the problem of the stabilization of periodic orbits of discrete-time dynamical system controlled by the PBC to the problem of leading the Jacobian of one point of the orbit to zero (matrix composed by zeros) with the simplification provided by Lemma 1 . The next step is to define a gain matrix $K_{k}\left(x_{k}\right)$ that leads to the desired result.

Note that the result shown in the Theorem 1 sets not only the eigenvalues of the monodromy matrix equal zero, but the entire matrix is equal to zero. This results in the cancellation of the linearized dynamics around the periodic orbit. Setting all the eigenvalues of the monodromy matrix to zero is characteristic of dead-beat controllers and leads do finite-time convergence for linear dynamics [Grasselli \& Lampariello, 1981]. However, applying this result, as we do here, to the linearized dynamics around the periodic orbit does not ensure finite-time convergence of the trajectories of the nonlinear system towards the latter.

\subsection{Stabilizing control laws: The invertible input matrix case}

Theorem 2. If the input matrix $\nabla_{u} f(k, x, u)$ is invertible for $x=x_{k}^{*}$ and $u=u_{k}\left(x_{k}^{*}\right)$ and the linear map that describes the evolution of a perturbation in the close vicinity of a trajectory of system (10) for $u_{k}\left(x_{k}\right)=0$ is hyperbolic, then there exists a control gain $K_{k}\left(x_{k}\right)$ that satisfies Theorem 1.

Proof. See Appendix A.

Invertible $\nabla_{u} f(k, x, u)$ matrix is typically the case for systems such that

$$
x_{k+1}=g\left(k, x_{k}\right)+u_{k},
$$

where $x$ and $u$ are vectors of the same dimension and the systems can be fully actuated.

The values of $K_{k}\left(x_{k}\right)$ selected in the sequel will be shown to fulfill Theorem 2 .

- Control law CL1. $K\left(x_{0}^{*}\right)$ is a constant matrix defined by:

$$
K\left(x_{0}^{*}\right)=-\left.\left.\left(\left.\nabla_{u} f\left(0, x_{0}^{*}, u\right)\right|_{u=u_{0}\left(x_{0}^{*}\right)}\right)^{-1} \nabla_{x} f\left(0, x, u_{0}\left(x_{0}^{*}\right)\right)\right|_{x=x_{0}^{*}}\left(\nabla_{x} \varphi(p, 0, x, 0)-I_{n}\right)^{-1}\right|_{x=x_{0}^{*}} .
$$

This results in a linear time-invariant control law whose determination also depends upon the UPO knowledge.

- Control law CL2. $K_{k}\left(x_{k}^{*}\right)$ is a time-varying matrix defined for each time $k \in \mathbb{Z}$ by:

$$
K_{k}\left(x_{k}^{*}\right)=-\left.\left.\left(\left.\nabla_{u} f\left(k, x_{k}^{*}, u\right)\right|_{u=u_{k}\left(x_{k}^{*}\right)}\right)^{-1} \nabla_{x} f\left(k, x, u_{k}\left(x_{k}^{*}\right)\right)\right|_{x=x_{k}^{*}}\left(\nabla_{x} \varphi(k+p, k, x, 0)-I_{n}\right)^{-1}\right|_{x=x_{k}^{*}},
$$

This results in a linear time-periodic control law whose determination depends also upon the UPO knowledge.

CL1 or CL2 require the exact knowledge of the UPO for the design of the control gain. A similar control law for dimension-1 discrete-time systems is applied in [Hino et al., 2002], here CL1 and CL2 are provided for $n$-dimensional systems.

Application of CL1 or CL2 necessitates to define which of the orbit points is the point $x_{0}^{*}$. A possible choice is to take $x_{0}^{*}$ as the point of the cycle minimizing the distance from $x_{0}$.

- Control law CL3. $K_{k}\left(x_{k}\right)$ is given as

$$
K_{k}\left(x_{k}\right)=-\left.\left.\left(\left.\nabla_{u} f\left(k, x_{k}, u\right)\right|_{u=u_{k}\left(x_{k}\right)}\right)^{-1} \nabla_{x} f\left(k, x, u_{k}\left(x_{k}\right)\right)\right|_{x=x_{k}}\left(\nabla_{x} \varphi(k+p, k, x, 0)-I_{n}\right)^{-1}\right|_{x=x_{k}} .
$$

Contrary to CL1 and CL2, the choice CL3 does not require any knowledge on the UPO (except the period $p)$. The calculus of $K_{k}\left(x_{k}\right)$ depends only on the actual state of the trajectory. 
One advantage of CL3 is the fact that it avoids the necessity of finding the UPO before stabilizing it. Another advantage is that, when designing the control gain for CL1 or CL2 (or any other control method that depends on UPO knowledge), errors in the UPO approximation lead to less accurate control gains. The PBC with CL3 can be applied to find (or refine, for inaccurate approximations) UPOs when using other control methods.

The three control laws satisfy the condition of Theorem 2, this implies that the proposed control laws result in the suppression of the linearised dynamics (perturbation) around the periodic orbit $\left(\Psi_{k}=0_{n}\right)$. This is done canceling the linearised dynamics at, at least, one point of the orbit $\left(\left.\nabla_{x} \psi\left(k, x, K_{k}\left(x_{k}^{*}\right)\right)\right|_{x=x_{k}^{*}}=0_{n}\right)$ and depends on the exact knowledge of $f\left(k, x_{k}, u_{k}\left(x_{k}\right)\right)$ for the calculation of $\nabla_{x} f\left(k, x, u_{k}\left(x_{k}\right)\right)$ and the future state. Model-plant mismatch results in $\Psi_{k} \neq 0_{n}$ and a brief robustness analysis of the control methods based on simulation is presented in Section 3.3.

\subsection{Stabilizing control laws: The non-invertible input matrix case}

The control laws proposed in Section 2.2 are limited to systems described by (1) that present an invertible input matrix $\nabla_{u} f\left(k, x_{k}, u\right)$. Most of the chaos control methods consider this matrix equal to the identity and it is omitted [Sanjuán \& Grebogi, 2010]. Although, in the control system literature it is called input matrix and models how control effort is applied to the state variables [Sontag, 1998]. Here we provide alternatives for a non-invertible $\nabla_{u} f\left(k, x_{k}, u\right): \mathbb{N} \times \mathbb{R}^{n} \times \mathbb{R}^{m} \rightarrow \mathbb{R}^{n \times m}$ with $K_{k}\left(x_{k}\right): \mathbb{N} \times \mathbb{R}^{n} \rightarrow \mathbb{R}^{m \times n}$, $m \leq n, m, n \in \mathbb{N}$, in the special case of single input systems, i.e., for $m=1$.

Note that condition (A.1) (required in the proof of Theorem 1, Appendix A) may be unsatisfiable for non-invertible $\nabla_{u} f\left(k, x_{k}, u\right)$ and the Theorem 1 is not applicable (Lemma 1 is still valid).

For the cases where the condition (A.1) can not be satisfied we provide the control law (17) below with a non-linear time-varying control gain $K_{k}\left(x_{k}\right)$ that may be applied making the Floquet multipliers of the controlled orbit equal to zero. The monodromy matrix is not necessarily $0_{n}$, but this control law is equivalent to CL3, in the sense that the Floquet multipliers are equal to 0, while no previous knowledge about the UPO is required. The main requirement to apply the result we are about to state (Theorem 3 below) is the existence of a given change of basis transforming, at any frozen time, the system linearised around the periodic orbit in its controllable canonical form. This condition is equivalent to controllability in linear time-invariant system theory [Sontag, 1998].

Theorem 3. Given the matrices

$$
\begin{aligned}
A_{k}\left(x_{k}\right) & =\left.\nabla_{x} f\left(k, x, u_{k}\left(x_{k}\right)\right)\right|_{x=x_{k}} \\
B_{k}\left(x_{k}\right) & =\left.\nabla_{u} f\left(k, x_{k}, u\right)\right|_{u=u_{k}\left(x_{k}\right)}
\end{aligned}
$$

associated to system (10) with scalar input signal $u_{k}\left(x_{k}\right)$, assume that:

(a) the unstable p-periodic orbit $x_{k}^{*}$ of system (1) is hyperbolic;

(b) for any $k \geq 0$ and for any $x_{k}$, the pair $\left(A_{k}\left(x_{k}\right), B_{k}\left(x_{k}\right)\right)$ is controllable;

(c) there exists a constant (with respect to $k$ ) transformation matrix $T$ that transforms $\left(A_{k}\left(x_{k}\right), B_{k}\left(x_{k}\right)\right.$ ) into the controllable canonical form $\left(T A_{k}\left(x_{k}\right) T^{-1}, T B_{k}\left(x_{k}\right)\right)$.

Then it is possible to set to zero all the Floquet multipliers of $x_{k}^{*}$ for system (10) using the time-varying control gain vector $K_{k}\left(x_{k}\right)$ defined by

$$
K_{k}\left(x_{k}\right)=
$$

where $N$ is the nilpotent matrix defined as

$$
N_{i, j}=\left\{\begin{array}{l}
1, \text { if } j=i+1 \\
0, \text { otherwise }
\end{array}\right.
$$

Proof. See Appendix A. 
Table 1. Points and respective values of the control gain for the stabilized UPO of Figure 1(a).

\begin{tabular}{|l||l|l|}
\hline Time & $k$ & $k+1$ \\
\hline$x_{k}^{*}$ & 0.90451 & 0.34549 \\
\hline$K_{k}\left(x_{k}^{*}\right)$ & -0.64721 & 0.24721 \\
\hline
\end{tabular}

\section{Numerical results}

This section is divided in four subsections. We compare the three control laws proposed for the PBC shown in the Section 2.2 using the Logistic map as case study in the first part (Section 3.1). We compare the PBC with the proposed CL3 with the DFC using as case study the Hénon map in the second part (Section 3.2). The DFC gain is tuned minimizing the modulus of the Floquet multipliers of the controlled orbits. A brief numerical robustness analysis of the PBC with CL3 and the DFC for the system subject to parametric uncertainty is done in the third part (Section 3.3). The fourth part is dedicated to the non-invertible input matrix case (Section 3.4).

\subsection{Comparison of the Prediction-Based Control laws}

The proposed control laws for the PBC are applied to the Logistic map. The proposed laws were developed for $n$-dimensional discrete-time systems, but a system of dimension $n=1$ simplifies the numerical analysis and the comparison among the three control laws.

\section{The Logistic map:}

The closed-loop Logistic map is given by

$$
x_{k+1}=g\left(x_{k}\right)+u_{k}\left(x_{k}\right),
$$

where $x: \mathbb{N} \rightarrow \mathbb{R}$ and $u: \mathbb{N} \times \mathbb{R} \rightarrow \mathbb{R}$. The function $g$ is given by

$$
g(x)=r x(1-x)
$$

for a given parameter $r \in \mathbb{R}$. The control signal is

$$
u_{k}(x)=K_{k}(x)(\varphi(k+p, k, x, 0)-x)
$$

where here $\varphi(k+p, k, x, 0)=g^{p}(x)$ and $K: \mathbb{N} \times \mathbb{R} \rightarrow \mathbb{R}$.

\subsubsection{Applying CL3 and finding UPOs}

The first step is applying CL3 because there is not the necessity of any knowledge about the target UPO position. We use CL3 to find the UPOs and CL1 and CL2 gains. Note that UPOs of the Logistic map can be found analytically, but CL3 simplifies the search.

CL3 applied to different values of the parameter $r$, initial condition $x_{0}$ and UPO period $p$ of the Logistic map is shown in Figure 1. In Figure 1(a) a 2-periodic orbit is stabilized for $r=4$ and initial condition $x_{0}=0.48$ resulting in $K_{k}\left(x_{k}^{*}\right)$ shown in Table 1. In Figure 1(b) a 3-periodic orbit is stabilized for $r=4$ and initial condition $x_{0}=0.69$ resulting in $K_{k}\left(x_{k}^{*}\right)$ shown in Table 2. In Figure 1(c) a 5-periodic orbit is stabilized for $r=4$ and initial condition $x_{0}=0.57$ resulting in $K_{k}\left(x_{k}^{*}\right)$ shown in Table 3 . In Figure $1(\mathrm{~d})$ a 6-periodic orbit is stabilized for $r=3.65$ and initial condition $x_{0}=0.52$ resulting in $K_{k}\left(x_{k}^{*}\right)$ shown in Table 4. The figure shows the evolution of the state $x_{k}$ and the control effort $u_{k}\left(x_{k}\right)$.

The first characteristic observed in the proposed scheme is the fast convergence of the trajectory to the vicinity of the target UPO. We consider that the UPO is stabilized when $\left|u_{k}\left(x_{k}\right)\right|<10^{-10}$, remaining below this threshold for the following $k$. The Figure 1(d) can be compared to [Morgül, 2009, Fig. 5], where the convergence is achieved for $k$ between 100 and 200 and using a larger control effort for the same conditions. 

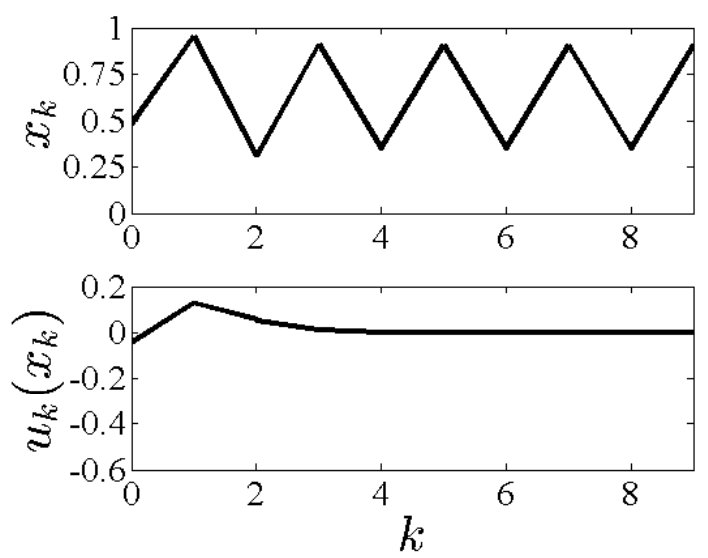

(a)
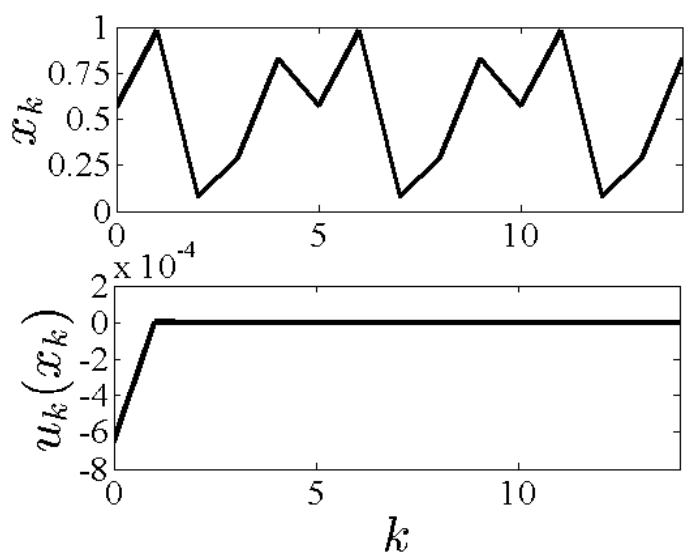

(c)
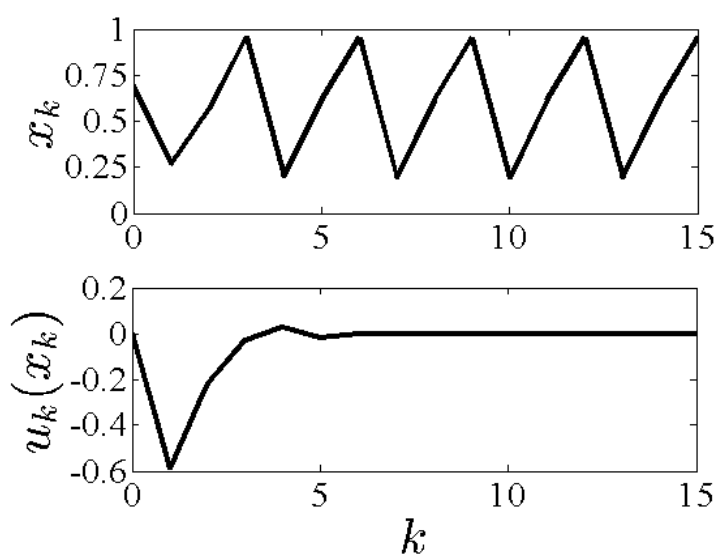

(b)
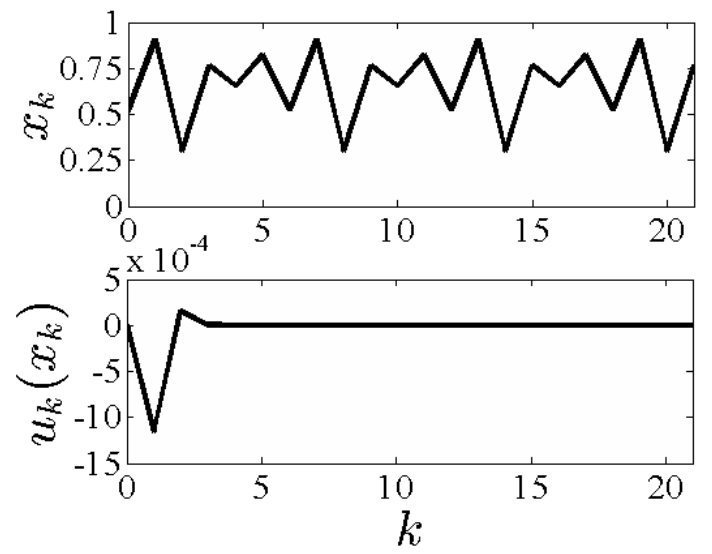

(d)

Fig. 1. Example of the evolution of the state $x_{k}$ and the control effort $u_{k}\left(x_{k}\right)$ for the Logistic map controlled using CL3: (a) $r=4, x_{0}=0.48$ and $p=2$; (b) $r=4, x_{0}=0.69$ and $p=3$; (c) $r=4, x_{0}=0.57$ and $p=5$; (d) $r=3.65, x_{0}=0.52$ and $p=6$.

Table 2. Points and respective values of the control gain for the stabilized UPO of Figure 1(b).

\begin{tabular}{|l||l|l|l|}
\hline Time & $k$ & $k+1$ & $k+2$ \\
\hline$x_{k}^{*}$ & 0.18826 & 0.61126 & 0.95048 \\
\hline$K_{k}\left(x_{k}^{*}\right)$ & -0.35628 & 0.12716 & 0.51484 \\
\hline
\end{tabular}

Table 3. Points and respective values of the control gain for the stabilized UPO of Figure 1(c).

\begin{tabular}{|l||l|l|l|l|l|}
\hline Time & $k$ & $k+1$ & $k+2$ & $k+3$ & $k+4$ \\
\hline$x_{k}^{*}$ & 0.57116 & 0.97975 & $0.79373 \times 10^{-1}$ & 0.29229 & 0.82743 \\
\hline$K_{k}\left(x_{k}^{*}\right)$ & $-0.17250 \times 10^{-1}$ & -0.11630 & 0.10197 & $0.50353 \times 10^{-1}$ & $-0.79377 \times 10^{-1}$ \\
\hline
\end{tabular}

Table 4. Points and respective values of the control gain for the stabilized UPO of Figure 1(d).

\begin{tabular}{|l||l|l|l|l|l|l|}
\hline Time & $k$ & $k+1$ & $k+2$ & $k+3$ & $k+4$ & $k+5$ \\
\hline$x_{k}^{*}$ & 0.90983 & 0.29944 & 0.76568 & 0.65486 & 0.82497 & 0.52704 \\
\hline$K_{k}\left(x_{k}^{*}\right)$ & -0.54423 & 0.26633 & -0.35281 & -0.20564 & -0.43154 & $-0.35909 \times 10^{-1}$ \\
\hline
\end{tabular}

\subsubsection{Comparing the three control laws by convergence rate and control effort}

The transients of $u_{k}\left(x_{k}\right)$ for trajectories converging to the target UPOs for the three control laws are compared on Figure 2. The examples refer only to stabilized orbits of period $p$, as there are control gains that make trajectories controlled by CL1 and CL2 diverge to infinity or converge to fixed points or periodic 
Table 5. Points and respective values of the control gain for one of the stabilized UPO of Figure 4.

\begin{tabular}{|l||l|l|l|}
\hline Time & $k$ & $k+1$ & $k+2$ \\
\hline$x_{k}^{*}$ & 0.41318 & 0.96985 & 0.11698 \\
\hline$K_{k}\left(x_{k}^{*}\right)$ & $0.77177 \times 10^{-1}$ & -0.41764 & 0.34046 \\
\hline
\end{tabular}

orbits that do not correspond to a solution of the free system (19) (situation that also occurs for the DFC $)^{1}$. In Figure 2(a) a 2-periodic orbit is stabilized for $x_{0}=0.48$, the control gain used for CL2 is the one obtained in the simulation of Figure 1(a) and the control gain used for CL1 is $K=0.24721$. In Figure 2(b) a 5-periodic orbit is stabilized for $x_{0}=0.6469$, the control gain used for CL2 is the one obtained in the simulation of Figure 1(c) and the control gain used for CL1 is $K=-0.17250 \times 10^{-1}$.

The trajectories shown in the Figure 2 make explicit the fact that, if the stabilization succeeds, the convergence for CL3 is faster than the convergence for the other two control laws. This is better evidenced for trajectories with initial conditions farther from the target UPO.

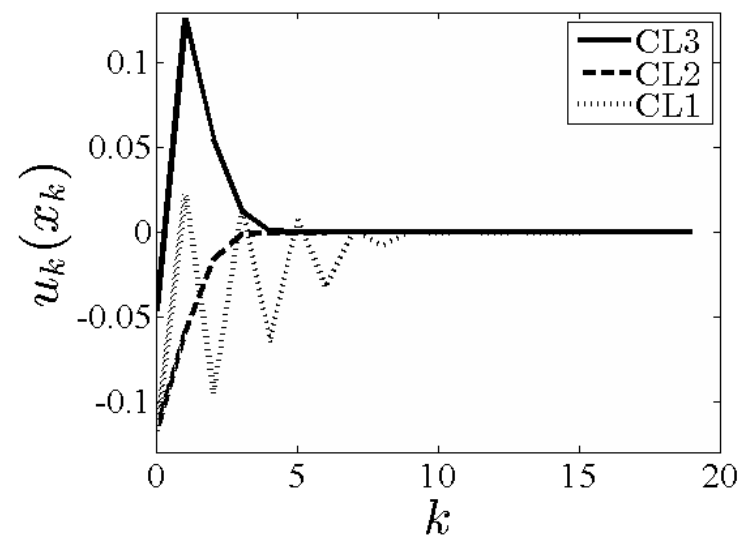

(a)

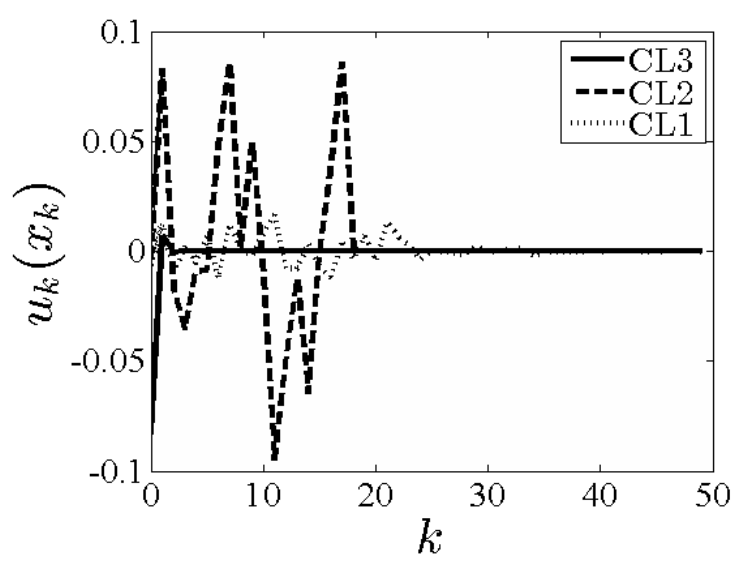

(b)

Fig. 2. Comparison among the control effort $u_{k}\left(x_{k}\right)$ transient of trajectories converging to stabilized orbits of the Logistic map using the three control laws (legend in the figure) for $r=4$ : (a) $x_{0}=0.48$ and $p=2$; (b) $x_{0}=0.6469$ and $p=5$.

\subsubsection{Comparing the three control laws by basins of attraction}

The comparison among the control laws is completed by the size of the basins of attraction (BA) of the stabilized orbits. The BAs here are the set of initial conditions that converge to a specific periodic orbit of the closed-loop system. The BAs of stabilized period-2 orbits are shown in the Figure 3 and period-3 are shown in the Figure 4. The different control laws are represented in the sub-figures (a), (b) or (c) of each figure. In Figures 3(c), 4(b) and 4(c), the BAs obtained for different control gains are divided in the vertical axis. The Logistic map has only one period-2 orbit for $r=4$ and the control gain on the orbit was obtained in the simulation of Figure 1(a). This results in two different gains for CL1. There exists two period-3 orbits for $r=4$ with two different values of $K_{k}\left(x_{k}^{*}\right)$ (two sets of control gains for CL2), the first is the one obtained in the simulation of Figure $1(\mathrm{~b})$ (Table 2) and the other is $K_{k}\left(x_{k}^{*}\right)$ shown in Table 5. This results in six different control gains for CL1.

The basins of attraction of the fixed points (FP: period- 1 orbits) are shown for $p=2$ and $p=3$ when using CL3, the other control laws do not stabilize fixed points when applying $p \neq 1$. The BAs of the orbit with period divisor of $p$ decrease in length for higher values of $p$. Another phenomenon observed when

\footnotetext{
${ }^{1}$ There may exist $p$-periodic orbits of the closed-loop system that are not $p$-periodic orbits of the free system. However, the occurrence of this phenomenon is detected when $u_{k}\left(x_{k}\right)$ does not converge to zero. This contrasts with the DFC, where $p$-periodic orbits of the closed-loop and the free system are exactly the same.
} 
increasing $p$ (see Figure 5) is that more orbits are stabilized, decreasing their individual BAs, but increasing the length of the set of the BAs of all stabilized orbits. This occurs due to the exponential growth of the UPO quantity by period and the local stability of the orbits achieved for the closed-loop system.

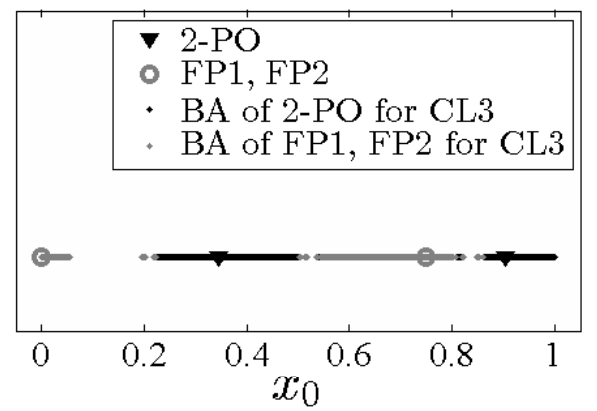

(a)

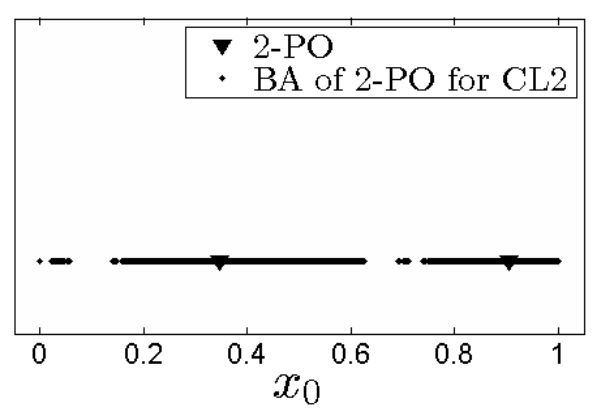

(b)

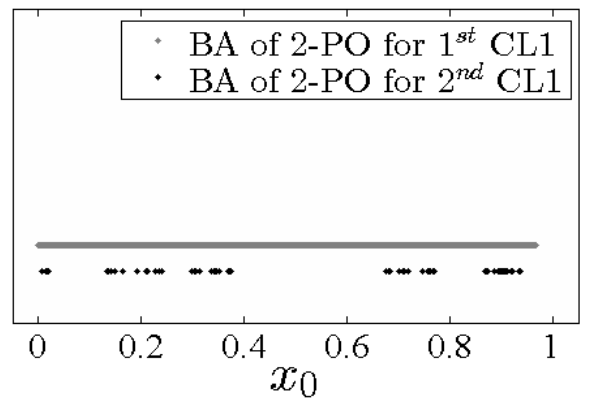

(c)

Fig. 3. Basins of attraction (BAs) of stabilized period-2 orbits of the Logistic map controlled with the three different control laws for $r=4$. FP1 and FP2 are fixed points and FP1, specifically, does not belong to the chaotic attractor.

The comparison of previous results shows that CL3 leads to faster convergence of trajectories to the target UPO while CL1 leads to slower convergence. The comparison of the BAs shows that CL3 leads to smaller BAs (when analysing a specific orbit) and different initial conditions may lead to different stabilized orbits. Specific values of the control gain for CL1 lead to the largest BAs. In both cases, CL2 leads to intermediate results. The greatest advantage of CL3 is that its application is much simpler than the others, it can be also used to find the UPOs or the stabilizing gains of the other control laws.

\subsection{Comparison of Prediction-Based and Delayed Feedback Control}

We now compare the PBC and DFC using the closed-loop Hénon map (22) as a case study. We chose CL3 because only the period of the UPO is needed for tuning the controller gain. We wish to reduce the implementation complexity finding the UPOs using CL3.

\section{The Hénon map:}

The closed-loop Hénon map is given by

$$
x_{k+1}=g\left(x_{k}\right)+u_{k}\left(x_{k}\right),
$$

where $x: \mathbb{N} \rightarrow \mathbb{R}^{2}$ and $u: \mathbb{N} \times \mathbb{R}^{2} \rightarrow \mathbb{R}^{2}$. The function $g$ is given by

$$
g\left(x_{k}\right)=\left[\begin{array}{c}
a-x_{1, k}^{2}+b x_{2, k} \\
x_{1, k}
\end{array}\right],
$$

for given parameters $a, b \in \mathbb{R}$. For the PBC we have

$$
u_{k}\left(x_{k}\right)=K_{k}\left(x_{k}\right)\left(\varphi\left(k+p, k, x_{k}, 0\right)-x_{k}\right),
$$




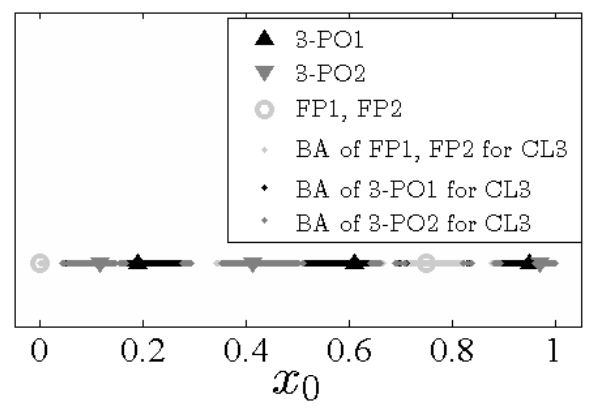

(a)

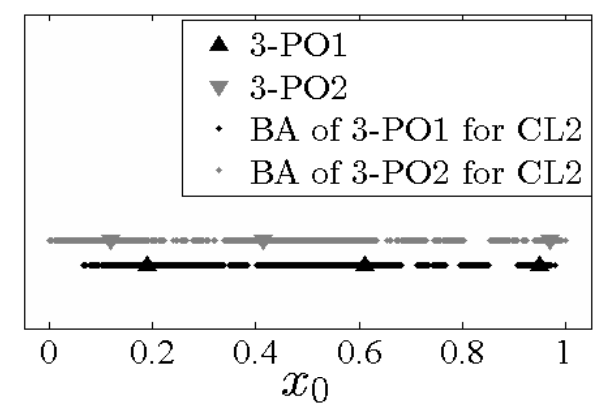

(b)

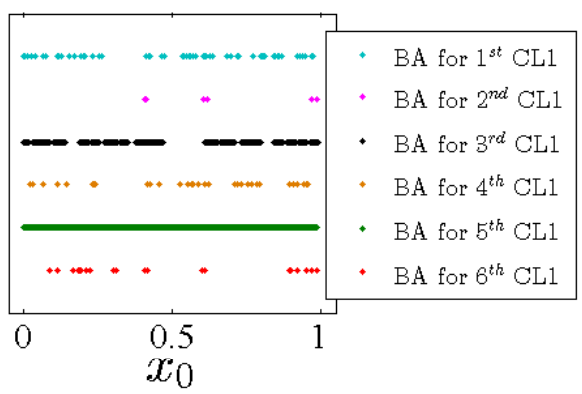

(c)

Fig. 4. Basins of attraction (BAs) of stabilized period-3 orbits of the Logistic map controlled with the three different control laws for $r=4$. FP1 and FP2 are fixed points and FP1, specifically, does not belong to the chaotic attractor.

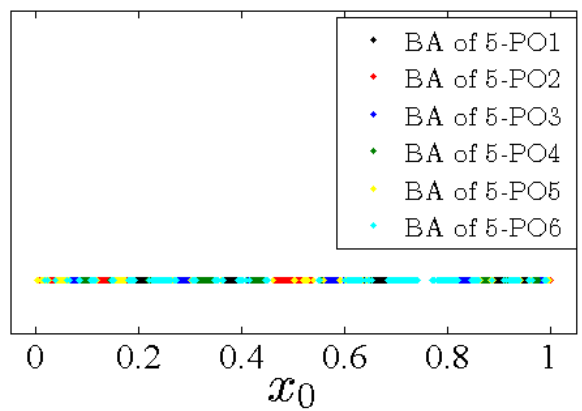

Fig. 5. Basins of attraction (BAs) of stabilized periodic orbits of the Logistic map using CL3 with $p=5$ and $r=4$.

where $\varphi(k+p, k, x, 0)=g^{p}(x)$ and $K: \mathbb{N} \times \mathbb{R}^{2} \rightarrow \mathbb{R}^{2 \times 2}$.

For the DFC we have

$$
u\left(x_{k}\right)=K\left(x_{k-p}-x_{k}\right),
$$

where $u: \mathbb{R}^{2} \times \mathbb{R}^{2}$ and $K \in \mathbb{R}^{2 \times 2}$.

We use the bifurcation digram of Figure 6 to identify chaos and its infinite number of UPOs. The diagram was generated plotting 500 points of $x_{1, k}$ after discarding the transient for $0 \leq k \leq 500$ using $b=0.3$ and $0 \leq a \leq 2$. A stable solution is not found for $a>1.428$. In the sequel $a=1.4$ and $b=0.3$.

\subsubsection{Applying CL3 and finding UPOs}

The first step is finding the target UPOs. This is a simple task when studying the Hénon map and these orbits can be easily found analytically. However, the PBC with CL3 can be used to systematize the process for this simple example or even more complex systems. It is possible to make a grid of initial conditions on 


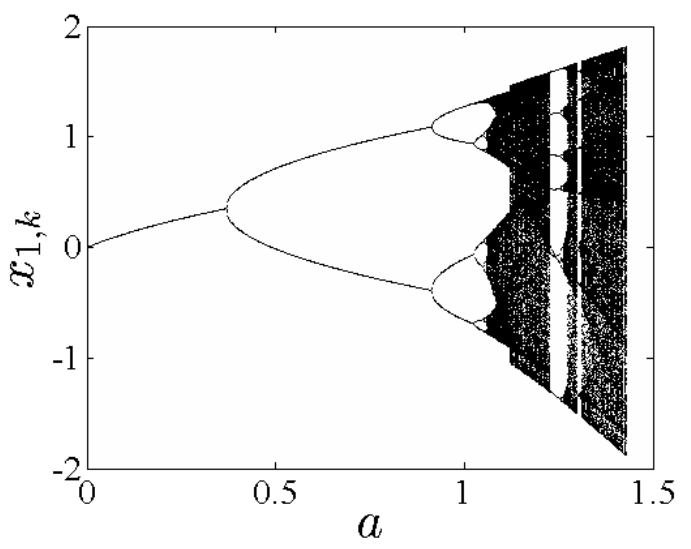

Fig. 6. Bifurcation diagram of the Hénon map for $b=0.3$.

Table 6. UPOs with period up to 6 of the Hénon map.

\begin{tabular}{|l||l|l|l|l|l|l|}
\hline UPO & FP1.1 & FP1.2 & P2 & P4 & P6.1 & P6.2 \\
\hline Period & 1 & 1 & 2 & 4 & 6 & 6 \\
\hline Free system & {$[-1.9237$,} & {$[3.2598,-$} & {$[-3.0101,-$} & {$[-8.6394,-$} & {$[-27.5147$,} & {$[28.1250$,} \\
eigenvalues & $0.1559]$ & $0.0920]$ & $0.0299]$ & $0.0009]$ & $\left.-2 \times 10^{-5}\right]$ & $\left.2 \times 10^{-5}\right]$ \\
\hline
\end{tabular}

Table 7. DFC control gain $K$; largest, in modulus, Floquet multipliers $\left(|\mu|_{\max }\right)$; Floquet multipliers $(\mu)$ of the stabilized orbits of the Hénon map.

\begin{tabular}{|c|c|c|c|}
\hline UPO & FP1.1 & P2 & $\overline{\mathrm{P} 4}$ \\
\hline$K$ & $\begin{array}{cc}-0.75420 & 0.01369 \\
-0.28878 & -0.00023\end{array}$ & $\begin{array}{lll}-0.16542 & 0.00675 \\
-1.98560 & 0.31435\end{array}$ & $\begin{array}{cc}-0.62885 & -0.18505 \\
0.19533 & -0.19424\end{array}$ \\
\hline$|\mu|_{\max }$ & 0.25346 & 0.5904 & 0.79702 \\
\hline$\mu$ & $-0.2534 \pm 0.0046 \mathrm{i}$ & $-0.5901 \pm 0.0190 \mathrm{i}$ & $-0.7954 \pm 0.0509 \mathrm{i}$ \\
\hline
\end{tabular}

the region of the state space that contains the chaotic set, apply CL3 for one value of $p$, for a large $k$ and for each initial condition, collect the points of the stabilized orbits and identify the period- $p$ orbits. The points of the identified orbits are used to design the control gains for the DFC.

A list of UPOs with period up to 6 is shown in the Table 6 with their period and eigenvalues for the free system. There is no orbits of period 3 and 5 for the chosen $a$ and $b$.

\subsubsection{Designing the DFC control gain by optimization}

The design of the control gain for the DFC is done by choosing a constant matrix $K$ that minimizes the largest, in modulus, Floquet multiplier $|\mu|_{\max }$ of the controlled orbit. The minimization is performed using the MATLAB ${ }^{\circledR}$ routine fminsearch that implements the Nelder-Med simplex direct-search method [Lagarias et al., 1998]. The initial condition of the elements of the matrix $K$ were scanned between -1 and 1. The matrix $\Psi_{k}$ and its eigenvalues were computed for each $K$ and the local minima of the largest eigenvalue in modulus were obtained (see Appendix B for the DFC monodromy matrix calculation). In Table 7 we summarise the best stabilizing $K$ and the respective largest eigenvalue of the controlled orbit and its modulus.

After several tests, adjusting the convergence parameters and initial conditions, no matrix gain was found that stabilizes the period-6 orbits and the fixed point FP1.2 with the DFC. It is not proved here that these orbits can not be stabilized with the DFC, but these results are in agreement with the literature, since orbits of higher periods and orbits with an odd number of real Floquet multipliers larger than +1 (odd-number limitation) are not stabilized by the DFC [Tian \& Zhu, 2004; Zhu \& Tian, 2005, 2008; Ushio, 1996]. Observing the Table 7 we see that $|\mu|_{\max }$ increases when increasing the period of the controlled orbit, resulting in $|\mu|_{\max }>1$ for the orbit P6.1. The orbits FP1.2 and P6.2 have one Floquet multiplier 
real and larger than +1 , characteristic of orbits originated from saddle-node bifurcations [Alligood et al., 1996]. The other orbits are originated by period-doubling bifurcations.

\subsubsection{Comparing PBC and DFC by basins of attraction}

The basis of attraction of the orbits controlled by the DFC are shown in the Figure 7. The initial condition of the delayed states was set on the target UPO, this results in 2 and 4 simulations for each basins of attraction for the orbits $\mathrm{P} 2$ and $\mathrm{P} 4$, respectively. The initial condition was set varying the initial point of the orbit and consequently the order of the points $x_{k-1} \ldots x_{k-p}$ in these cases. The basin of attraction of the orbit $\mathrm{P} 4$, locally stable when controlled by the DFC, is not shown here. A scan with a step of 0.005 in $x_{1,0}$ and $x_{2,0}$ was not sufficient to find a point that converges to the orbit and we conclude that its basin of attraction is limited to a very small vicinity of the orbit. In the Figure 7(b), the colours blue and green were used to separate the BA of the P2 in two parts, according to the initial condition of the delayed states.

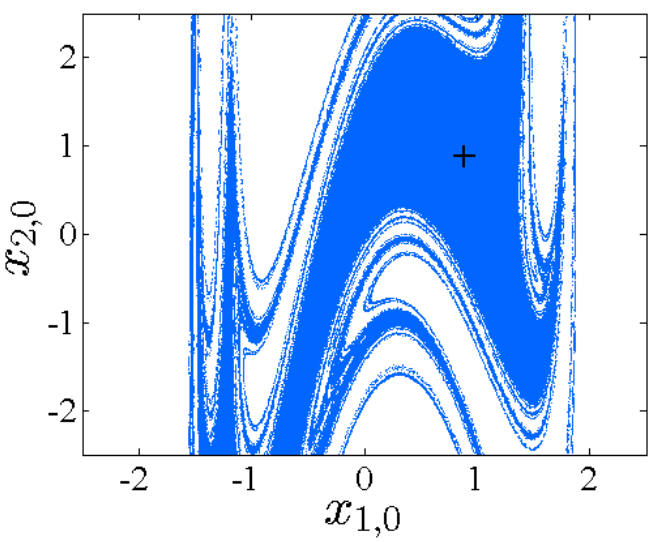

(a)

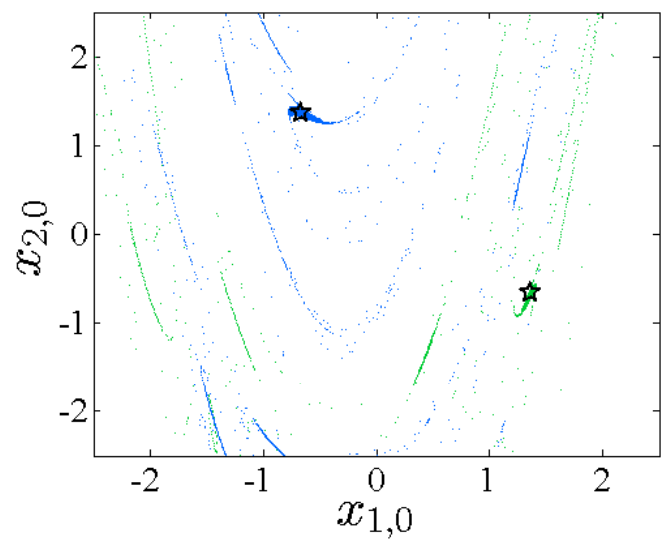

(b)

Fig. 7. Basins of attraction of the orbits controlled by the DFC for the Hénon map. (a) FP1.1 (+), (b) P2 (*)

The basins of attraction of the orbits controlled by the PBC are shown in the Figure 8 . We observe that all the orbits of period $p$ and its divisors are stabilized for the same value of $p$ used in the control laws. This results in more than one BA represented in each figure. The basins of FP1.1 and FP1.2 are also shown in the Figures $8(\mathrm{~b})$ and $8(\mathrm{c})$, this suggests that the basins of the orbits with period divisor of $p$ reduce the size when increasing $p$. The basin of the orbit P2 was not included in the Figure 8(c), the same choice was adopted for the BAs of the orbits FP1.1, FP1.2 and P2 in the Figure 8(d). The orbit FP1.2 does not pertain to the chaotic attractor of the Hénon map for the chosen $a$ and $b$, however it was also stabilized.

Comparing Figures 7 and 8 we observe that the PBC with the proposed control law not only stabilizes orbits that the DFC does not stabilize, but also leads to larger basins of attractions. Although, as verified for the Logistic map when applying CL3, different orbits are stabilized with the PBC, the orbits of period- $p$ and its divisors.

A characteristic better observed in this bi-dimensional example (notably in Figures 8(c) and 8(d)) is the apparent fractal boundary between the basins [Alligood et al., 1996].

\subsubsection{Comparing PBC and DFC by convergence rate and control effort}

Figure 9 shows the sum of the modulus of the control effort in both directions to stabilize the orbits $\mathrm{P} 2$ and P4 using the DFC and the PBC. The vertical axis is in logarithmic scale to better compare the convergence rate to the UPO using each method, the control effort is represented for $\left\|u_{k}\left(x_{k}\right)\right\|_{1}>10^{-10}$, $\|\cdot\|_{1}$ is the norm-1. The data corresponding to the DFC in the Figure $9(\mathrm{~b})$ are plotted at each ten points. The convergence rate of the trajectory in both cases is faster for the PBC, this happens even with the extended states of the DFC initially set on the target UPO and using as $x_{k-1}$ the point of $x_{k}^{*}$ closer to $x_{0}$. 


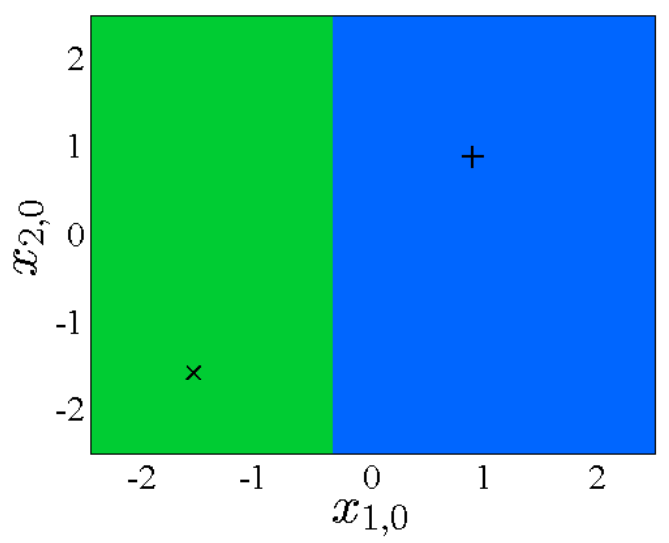

(a)

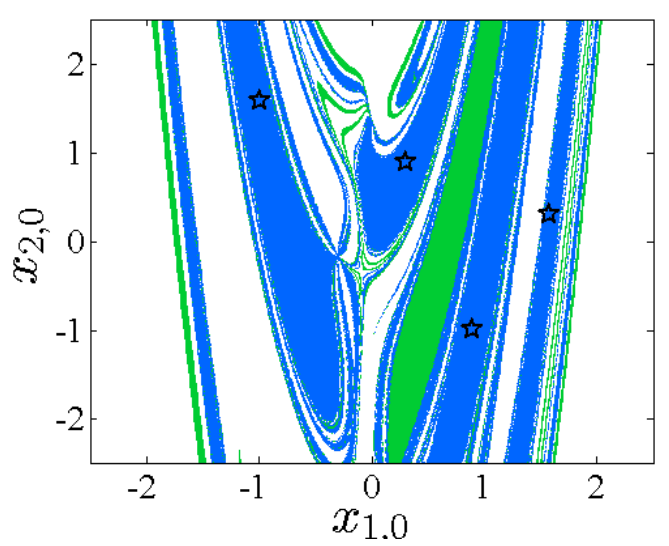

(c)

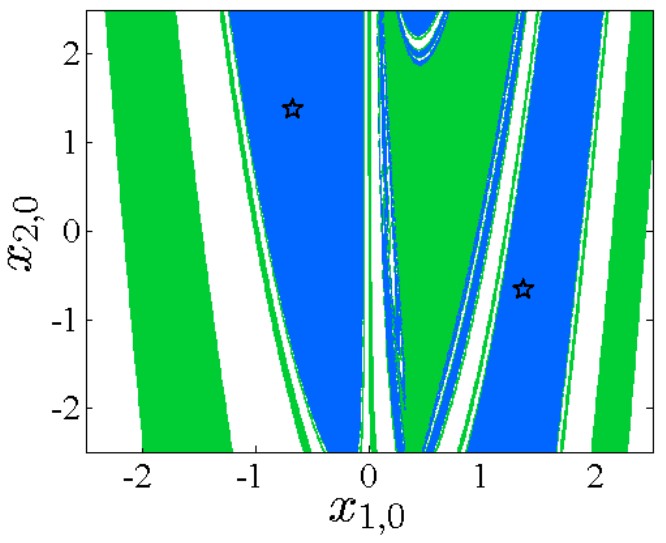

(b)

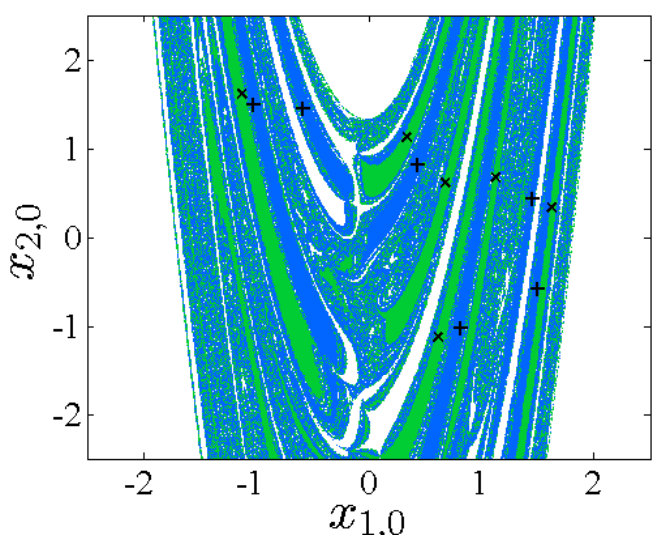

(d)

Fig. 8. Basins of attraction of the orbits controlled by the PBC for the Hénon map. (a) $p=1$, FP1.1 (+) and its BA blue and FP1.2 $(\times)$ and its BA in green; (b) $p=2, \mathrm{P} 2(\star)$ and its BA in blue and the BAs of the fixed points in green; (c) $p=4$, $\mathrm{P} 4(\star)$ and its BA in blue and the BAs of the fixed points in green; $(\mathrm{d}) p=6, \mathrm{P} 6.1(\times)$ and its BA in green, P6.2(+) and its $\mathrm{BA}$ in blue.

The trajectory controlled with the PBC presents lower control effort amplitude compared to the DFC for the tests performed.

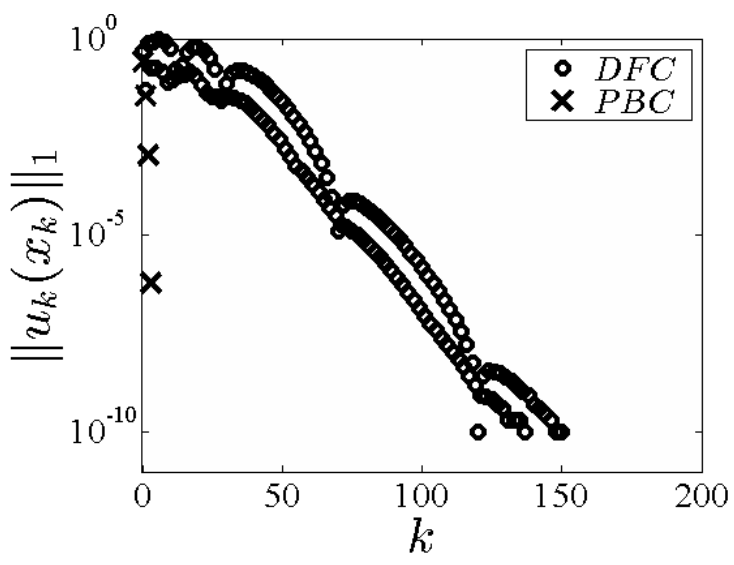

(a)

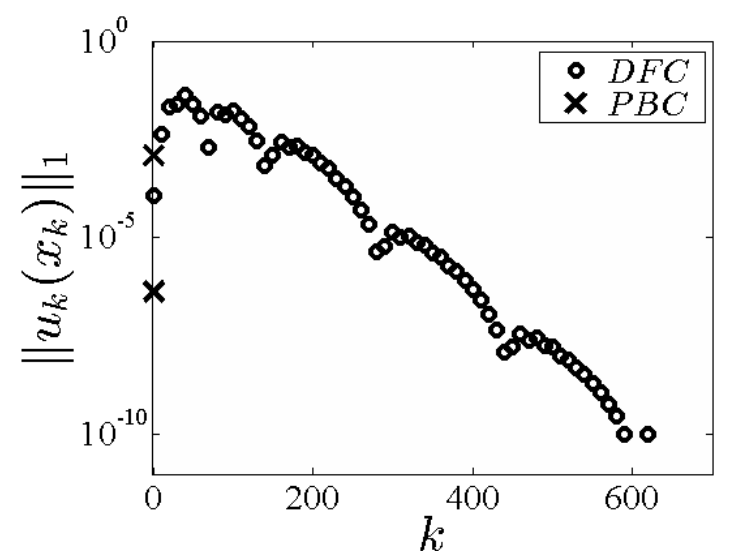

(b)

Fig. 9. Time series of the control effort applied to stabilize periodic orbits of the Hénon map using the DFC and PBC. (a) $p=2, x_{0}=[-0.5 ; 1]$; (b) $p=4, x_{0}=[0.305 ; 0.893]$. 
We verified that the PBC does not present the odd-number limitation and can stabilize orbits with longer periods; both are known DFC limitations [Zhu \& Tian, 2005]. The basins of attraction and the convergence rate of the trajectories of the orbits stabilized by the PBC are larger than for the DFC, with a lower control effort.

The PBC depends on a free system prediction model when applied, but the DFC can be applied without model, it is only necessary to record the delayed states. This characteristic favours the DFC, but its control gain design depends on a model and on the target UPO for an analytical or numerical tuning. The PBC with the proposed control law has the advantage of being independent of previous knowledge about the target UPO position and it is useful for applications where the orbit is unknown.

The choice of the cost function for the optimization of the DFC control gain favours the local stability of the controlled orbit, however it does not guarantee a maximum for the basin of attraction size. The possibility of better results than the ones presented in the comparison between methods is not to be excluded.

\subsection{A brief robustness analysis on the prediction-based control}

Here we evaluate the robustness of the PBC using CL3 for a system subjected to parametric uncertainties and compare the results for the DFC under the same conditions.

\subsubsection{Defining the uncertainties}

\section{The PBC case:}

For the PBC we consider a parametric error between the real free system $f\left(k, x_{k}, 0\right)$ and the free system prediction model, here named $\hat{f}\left(k, x_{k}, 0\right)$. In the sequel, $\hat{\cdot}$ refers to the prediction model. We apply CL3 on the closed-loop system (10) and use the notation

$$
\psi(k, x, K) \doteq f\left(k, x, u_{k}(x)\right), \quad u_{k}(x)=K(\hat{\varphi}(k+p, k, x, 0)-x),
$$

where $\hat{\varphi}(k+p, k, x, 0)$ is defined for $\hat{f}(k, x, 0)$.

Note that the Lemma 1 is not (necessarily) valid now because $x_{k}^{*}$ is not (necessarily) a periodic orbit of $\psi\left(k, x_{k}, K_{k}\left(x_{k}\right)\right)$ with $u_{k}\left(x_{k}\right)$ defined in $(26)$, namely $\hat{x}_{k}^{*}$. The monodromy matrix of this new orbit is given by

$$
\Psi_{k}=\left.\prod_{l=0}^{p-1} \nabla_{x} \psi\left(k+l, x, K_{k+l}(x)\right)\right|_{x=\hat{x}^{*}(k+l)},
$$

with

$$
\begin{array}{r}
\nabla_{x} \psi\left(k, x, K_{k}(x)\right)=\nabla_{x} f\left(k, x, u_{k}\left(x_{k}\right)\right)+\nabla_{u} \hat{f}\left(k, x_{k}, u\right) K_{k}\left(x_{k}\right) \nabla_{x}(\hat{\varphi}(k+p, k, x, 0)-x)+ \\
\nabla_{u} \hat{f}\left(k, x_{k}, u\right) \nabla_{x} K_{k}(x)\left(\hat{\varphi}\left(k+p, k, x_{k}, 0\right)-x_{k}\right) .
\end{array}
$$

\section{The DFC case:}

For the DFC we use

$$
u_{k}\left(x_{k}\right)=K\left(x(k-p)-x_{k}\right)
$$

where $K$ is the optimal gain used to stabilize $\hat{x}_{k}^{*}$.

\subsubsection{Comparing $P B C$ and $D F C$}

The robustness analysis is performed using the Hénon map (22) as case study for the comparison between methods.

We define $\hat{a}=1.4$ and $\hat{b}=0.3$ for $\hat{f}\left(k, x_{k}, 0\right), b=0.3$ and vary $a$ for $f\left(k, x_{k}, 0\right)$. We try to stabilize the orbit P2 with both methods for $0.91<a<2$, where the limit $a=0.91$ refers to the bifurcation that originates the UPO P2 and $a=2$ was used to apply the control schemes in a system without stable solutions. Here, $\nabla_{u} \hat{f}\left(k, x_{k}, u\right)=\nabla_{u} f\left(k, x_{k}, u\right)=B=I_{n}$. 


\section{Comparison criteria:}

The comparison is performed using the maximum, in modulus, Floquet multiplier $|\mu|_{\max }$ of the controlled orbit, $\hat{x}_{k}^{*}$, and the control effort for one cycle of the steady state trajectory, $v$, defined as

$$
v=\lim _{k \rightarrow+\infty} \sum_{l=1}^{p}\left\|u_{k+l}\left(x_{k+l}\right)\right\|_{1}
$$

$\|\cdot\|_{1}$ is the norm-1 used to measure the total external effort necessary to stabilization.

\section{Results and analysis:}

The results are shown in Figure 10 and $\hat{x}_{1}^{*}$ is shown in Figure 11 using as initial condition the points of P2 for $a=1.4$, including the delayed states for the DFC.

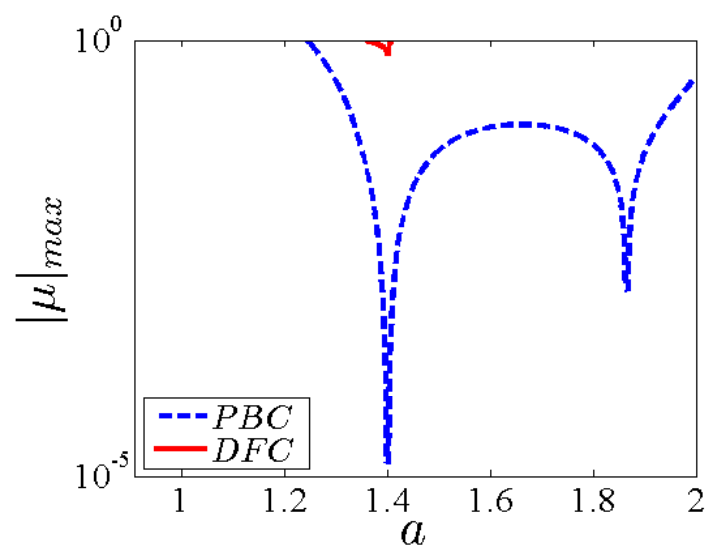

(a)

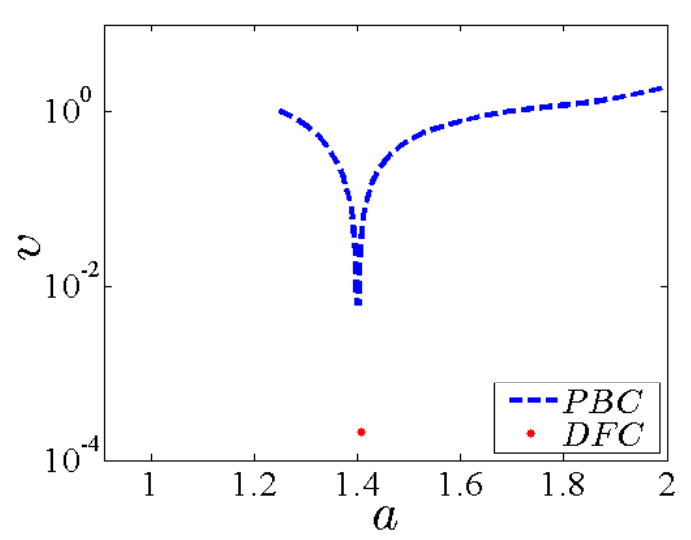

(b)

Fig. 10. Comparison between the PBC with CL3 and the DFC stabilizing the $\hat{x}_{k}^{*}$ for $b=0.3$. (a) the maximum, in modulus, Floquet multiplier; (b) the control effort for one cycle of the steady state system.

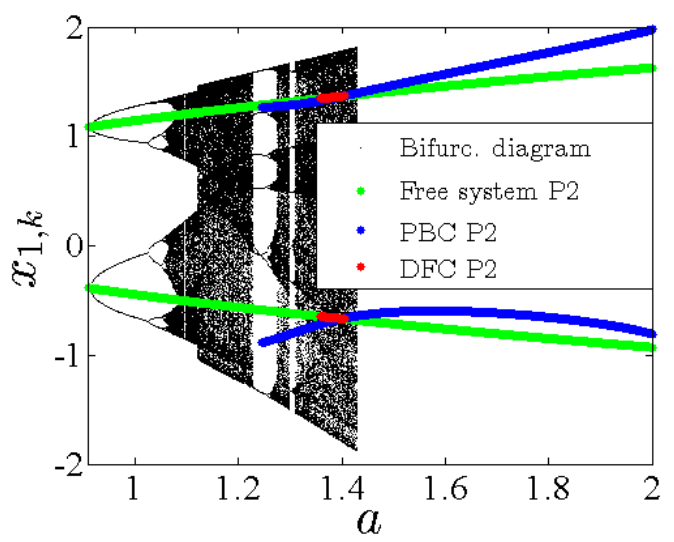

Fig. 11. Stabilized $\hat{x}_{k}^{*}$ using the PBC (blue) and the DFC (red) on the bifurcation diagram (black) of the Hénon map for $b=0.3$. The orbit $x_{k}^{*}$ of the free system is in green

Figure 10(a) shows that the orbit controlled by the PBC is more stable than the orbit controlled by the DFC. For $a=\hat{a}=1.4$, as expected, $|\mu|_{\max } \approx 0$ for the PBC and this point is not represented in the figure due to the logarithmic scale. 
Figure 10(b) shows that the orbit controlled by the DFC presents a steady state control effort approximately equal to zero (not shown due to logarithm scale). The point in red shown in the figure is obtained for a value of $a$ where $|\mu|_{\max } \approx 1$ and the convergence is slow. For the $\mathrm{PBC}$ we have $v \approx 0$ for $a=\hat{a}=1.4$ and a larger control effort for other values.

Figure 11 shows the stabilized orbit for both control methods. The analysis of the Figures 10 and 11 allows to conclude that PBC method is applicable for a larger interval of $a$, including values where there is not a stable solution for the free system $(a>1.428)$, the controlled orbit is more stable, but the control effort is larger in comparison with the DFC. The choice of the method to be used depends on the performance criteria of the control problem.

\subsection{Prediction-based control for non-invertible input matrix}

Here we use the PBC to stabilize periodic orbits of the Hénon map (22) for a non-invertible input matrix $\nabla_{u} f\left(k, x_{k}, u\right)=B$ with a control law similar to CL3 with no need of previous knowledge about the UPO position.

Here, the matrices $B$ and $K_{k}\left(x_{k}\right)$ are given by

$$
B=\left[\begin{array}{l}
1 \\
0
\end{array}\right], \quad K_{k}\left(x_{k}\right)=\left[k_{1, k}\left(x_{k}\right) k_{2, k}\left(x_{k}\right)\right] .
$$

In this case we have a scalar control signal $(7), u: \mathbb{N} \times \mathbb{R}^{2} \rightarrow \mathbb{R}$.

The matrix $\nabla_{x} f\left(k, x, u_{k}\left(x_{k}\right)\right)$ for the Hénon map is

$$
\nabla_{x} f\left(k, x, u_{k}\left(x_{k}\right)\right)=\left[\begin{array}{cc}
-2 x_{1, k} & b \\
1 & 0
\end{array}\right]
$$

and the matrix $\nabla_{x} \varphi(k+p, k, x, 0)$ is written as

$$
\nabla_{x} \varphi(k+p, k, x, 0)=\left[\begin{array}{l}
f p_{11, k}\left(x_{k}\right) f p_{12, k}\left(x_{k}\right) \\
f p_{21, k}\left(x_{k}\right) f p_{22, k}\left(x_{k}\right)
\end{array}\right]
$$

Solving (17) for

$$
T=\left[\begin{array}{ll}
0 & 1 \\
1 & 0
\end{array}\right]
$$

we obtain

$$
\begin{aligned}
k_{1, k}\left(x_{k}\right) & =\frac{-b f p_{21, k}\left(x_{k}\right)+2 x_{1, k}-2 f p_{22, k}\left(x_{k}\right) x_{1, k}}{f p_{11, k}\left(x_{k}\right)+f p_{22, k}\left(x_{k}\right)-f p_{11, k}\left(x_{k}\right) f p_{22, k}\left(x_{k}\right)+f p_{12, k}\left(x_{k}\right) f p_{21, k}\left(x_{k}\right)-1} \\
k_{2, k}\left(x_{k}\right) & =\frac{b f p_{11, k}\left(x_{k}\right)+2 f p_{12, k}\left(x_{k}\right) x_{1, k}-b}{f p_{11, k}\left(x_{k}\right)+f p_{22, k}\left(x_{k}\right)-f p_{11, k}\left(x_{k}\right) f p_{22, k}\left(x_{k}\right)+f p_{12, k}\left(x_{k}\right) f p_{21, k}\left(x_{k}\right)-1} .
\end{aligned}
$$

In this case, the matrices $\nabla_{x} f\left(k, x, u_{k}\left(x_{k}\right)\right)$ and $\nabla_{u} f\left(k, x_{k}, u\right)$ are already in a controllable canonical form. This allows to obtain a constant $T$ and a matrix $K_{k}\left(x_{k}\right)$. For $B=\left[\begin{array}{ll}0 & 1\end{array}\right]^{\prime}, T_{k}\left(x_{k}\right)$ is not constant and it is necessary to calculate a control gain using the previous knowledge of $x_{k}^{*}$, similar to CL1 and CL2.

A numerical example is shown in the Figure 12. This example can be compared to the results of Figure 9 showing that the convergence rate for the PBC applied for a non-invertible input matrix case has the same magnitude of the convergence rate for the invertible input matrix case. It is also faster than the DFC applied to the invertible input matrix case.

\section{Conclusions}

New control laws for the PBC were presented here. The new control laws were compared numerically among them and the most interesting one (CL3) was compared with the DFC presenting better results. This new control laws were formulated merging the chaos control and modern control theories. The problem of 

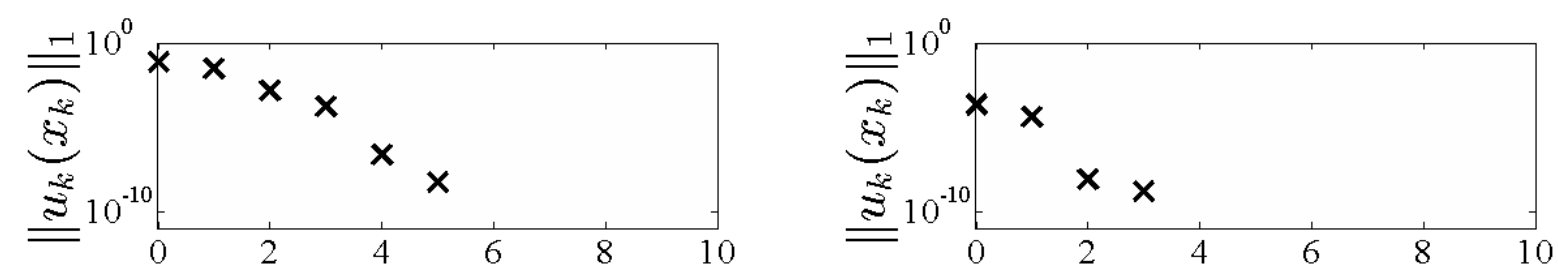

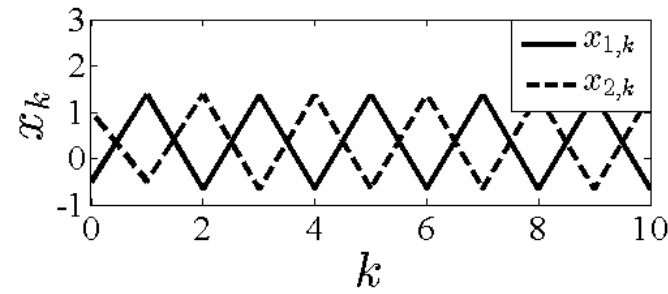

(a)

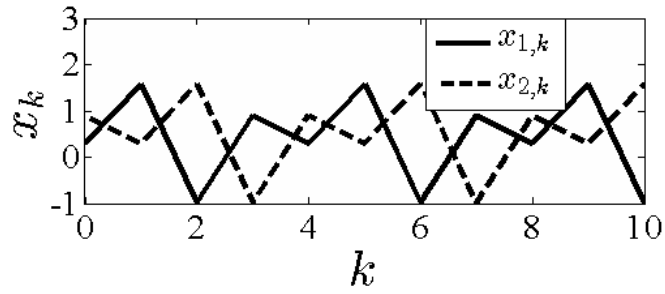

(b)

Fig. 12. Time series of the control effort and state variables of stabilized periodic orbits of the Hénon map using the PBC for a non-invertible input matrix $B=[10]^{\prime}$. (a) $p=2, x_{0}=[-0.5 ; 1]$; (b) $p=4, x_{0}=[0.305 ; 0.893]$.

stabilizing periodic orbits of nonlinear discrete-time systems was approximated locally as stabilizing linear time-periodic discrete-time systems resulting in a dead-beat like controller.

The new control law CL3 proposed has some practical advantages once it does not require the UPO position, can be rearranged for systems that are not fully actuated and is more robust to model parametric uncertainties than the classical DFC.

Analytical formulation and numerical results are sufficient for the proposition of the method and its practical advantages, although, experiments should be performed to complete the analysis. Propositions of these experiments are encouraged by the authors and have been analyzed for future research.

\section{Appendix A}

\section{Appendix A: Proofs}

Proof. [Proof of Lemma 1] The monodromy matrix of the closed-loop system is calculated as follows,

$$
\Psi_{k}=\left.\prod_{l=0}^{p-1} \nabla_{x} \psi\left(k+l, x, K_{k+l}(x)\right)\right|_{x=x_{k+l}^{*}} .
$$

Now, using the definition of $\psi(k, x, K),(11)$, we compute the derivative using the general chain rule

$$
\begin{array}{r}
\nabla_{x} \psi\left(k, x, K_{k}(x)\right)=\nabla_{x} f\left(k, x, u_{k}\left(x_{k}\right)\right)+\nabla_{u} f\left(k, x_{k}, u\right) K_{k}\left(x_{k}\right) \nabla_{x}(\varphi(k+p, k, x, 0)-x)+ \\
\nabla_{u} f\left(k, x_{k}, u\right) \nabla_{x} K_{k}(x)\left(\varphi\left(k+p, k, x_{k}, 0\right)-x_{k}\right)
\end{array}
$$

and apply $x=x_{k}=x_{k}^{*}$, resulting in

$$
\begin{aligned}
\left.\nabla_{x} \psi\left(k, x, K_{k}(x)\right)\right|_{x=x_{k}^{*}}= & \left.\nabla_{x} \psi\left(k, x, K_{k}\left(x_{k}^{*}\right)\right)\right|_{x=x_{k}^{*}}+ \\
& \left.\nabla_{u} f\left(k, x_{k}^{*}, u\right) \nabla_{x} K_{k}(x)\right|_{x=x_{k}^{*}}\left(\varphi\left(k+p, k, x_{k}^{*}, 0\right)-x_{k}^{*}\right) .
\end{aligned}
$$

On the periodic orbit, we have $\varphi\left(k+p, k, x_{k}^{*}, 0\right)=x_{k}^{*}$, and the term containing $\nabla_{x} K_{k}(x)$ is zero. This provides the desired result.

Observe that, for any $k \in \mathbb{Z}$, the $(i, j)$-th component of the product of the tensor $\left.\frac{\partial K_{k}(x)}{\partial x}\right|_{x=x_{k}^{*}}$ by the 
vector $\left(\varphi\left(k+p, k, x_{k}^{*}, 0\right)-x_{k}^{*}\right)$ can be computed using the following sum

$$
\sum_{j^{\prime}=1}^{n}\left(\left.\frac{\partial K_{k, i j^{\prime}}(x)}{\partial x_{j}}\right|_{x=x_{k}^{*}} \cdot\left(\varphi\left(k+p, k, x_{k}^{*}, 0\right)-x_{k}^{*}\right)_{j^{\prime}}\right) .
$$

This sum illustrates the dimensional consistence on the matrices multiplications shown in the proof of the lemma.

Proof. [Proof of Theorem 1] The proof is obtained by direct observation of the result in Lemma 1: under the conditions of the statement, $\Psi_{k}=0_{n}$, which yields stability of the associated fixed point, and thus stability of the periodic cycle.

Proof. [Proof of Theorem 2]

From Lemma 1,

$$
\left.\nabla_{x} \psi\left(k, x, K_{k}(x)\right)\right|_{x=x_{k}^{*}}=0_{n}
$$

is equivalent to

$$
\left.\nabla_{x} f\left(k, x, u_{k}\left(x_{k}^{*}\right)\right)\right|_{x=x_{k}^{*}}+\left[\nabla_{u} f(k, x, u) K_{k}(x)\left(\nabla_{x} \varphi(k+p, k, x, 0)-I_{n}\right)\right]_{x=x_{k}^{*}, u=u_{k}\left(x_{k}^{*}\right)}=0_{n} .
$$

If $\nabla_{u} f(k, x, u)$ and $\left(\nabla_{x} \varphi(k+p, k, x, 0)-I_{n}\right)$ are invertible for $x=x_{k}^{*}$ and $u=u_{k}\left(x_{k}^{*}\right), K_{k}\left(x_{k}\right)$ can be isolated in the right side of (A.1). This is the case if $\nabla_{x} \varphi(k+p, k, x, 0)$ is hyperbolic (eigenvalues different from one).

Proof. [Proof of Theorem 3] Using Lemma 1 the Jacobian matrix of system 1 subjected to (11) in the controllable canonical form is calculated as

$$
\left.\nabla_{x} \psi\left(k, x, k_{k}\left(x_{k}^{*}\right)\right)\right|_{x=x_{k}^{*}}=T\left[A_{k}\left(x_{k}^{*}\right)+B_{k}\left(x_{k}^{*}\right) K_{k}\left(x_{k}^{*}\right)\left(\left.\nabla_{x} \varphi(k+p, k, x, 0)\right|_{x=x_{k}^{*}}-I_{n}\right)\right] T^{-1} .
$$

and for each time $k=0,1, \ldots, p$ we want to guarantee

$$
T\left[A_{k}\left(x_{k}^{*}\right)+B_{k}\left(x_{k}^{*}\right) K_{k}\left(x_{k}^{*}\right)\left(\left.\nabla_{x} \varphi(k+p, k, x, 0)\right|_{x=x_{k}^{*}}-I_{n}\right)\right] T^{-1}=N
$$

where $N \in \mathbb{R}^{n \times n}$ is the nilpotent matrix (18). If we manage to realize (A.2), then from (12) we have that

$$
\Psi_{k}=\prod_{0}^{p-1} T^{-1} N T=T^{-1} N^{p} T
$$

has all its eigenvalues equal to zero due to similarity between $N$ and $T^{-1} N T$. For $p \geq n$, we obtain the special case $\Psi_{k}=0_{n}$.

We attempt now to solve equation (A.2) for the gain value $K_{k}\left(x_{k}^{*}\right)$. From (A.2) we deduce

$$
T B_{k}\left(x_{k}^{*}\right) K_{k}\left(x_{k}^{*}\right)=\left(N-T A_{k}\left(x_{k}^{*}\right) T^{-1}\right) T\left(\left.\nabla_{x} \varphi(k+p, k, x, 0)\right|_{x=x_{k}^{*}}-I_{n}\right)^{-1}
$$

where, using the fact that the pair $\left(T A_{k}\left(x_{k}\right) T^{-1}, T B_{k}\left(x_{k}\right)\right)$ is in the controllable canonical form and we are using scalar input function, one gets

$$
T B_{k}\left(x_{k}^{*}\right) K_{k}\left(x_{k}^{*}\right)=\left[\begin{array}{c}
0_{(n-1) \times n} \\
K_{k}\left(x_{k}^{*}\right)
\end{array}\right] .
$$

The defined $N$ implies that $\left(N-T A_{k}\left(x_{k}^{*}\right) T^{-1}\right)$ has the first $n-1$ lines equal to $0_{(n-1) \times n}$ guaranteeing the identity for the $(n-1)$ first lines of (A.4).

From (18), (A.4) and (A.5) we obtain

$$
K_{k}\left(x_{k}^{*}\right)=\left[0_{1 \times n-1} 1\right]\left(T^{-1} N T-A_{k}\left(x_{k}^{*}\right)\right)\left(\left.\nabla_{x} \varphi(k+p, k, x, 0)\right|_{x=x_{k}^{*}}-I_{n}\right)^{-1} .
$$

Notice that: 
- Applying (A.6) for $k \geq 0$ at any state $x_{k}$ we have (17) and local stability of $x_{k}^{*}$ is guaranteed by (A.2) and (A.3);

- If it is not possible to obtain a constant matrix $T$ then (A.3) is not verified and zero eigenvalues are not guaranteed. However stability of $x_{k}^{*}$ may be achieved by using others control laws.

Once the controllable canonical form is not unique, here consider it as following shown for linear time-invariant systems (for simplicity)

$$
\begin{gathered}
z_{k+1}=A_{c} z_{k}+B_{c} u_{k} \\
A_{c}=\left[\begin{array}{cccccc}
0 & 1 & 0 & 0 & \cdots & 0 \\
0 & 0 & 1 & 0 & \cdots & 0 \\
0 & 0 & 0 & 1 & \cdots & 0 \\
\vdots & \vdots & \vdots & \vdots & \ddots & \vdots \\
0 & 0 & 0 & 0 & \cdots & 1 \\
-a_{1}-a_{2} & -a_{3} & -a_{4} & \cdots & -a_{n}
\end{array}\right] \quad B_{c}=\left[\begin{array}{c}
0 \\
0 \\
0 \\
\vdots \\
0 \\
1
\end{array}\right] .
\end{gathered}
$$

\section{Appendix B: Monodromy matrix for discrete-time delayed feedback control}

In this appendix we provide the equations to calculate the monodromy matrix for the system controlled by the DFC with a constant gain matrix $K$ (A.8).

$$
x_{k+1}=f\left(k, x_{k}, K\left(x_{k-p}-x_{k}\right)\right)
$$

$x: \mathbb{N} \rightarrow \mathbb{R}^{n}, K \in \mathbb{R}^{n \times n}, f: \mathbb{N} \times \mathbb{R}^{n} \times \mathbb{R}^{n} \rightarrow \mathbb{R}^{n}$ and $k, p, n \in \mathbb{N}$.

The state vector $x_{k}$ is not sufficient to represent the dynamics of the closed-loop system with the DFC. We define an extended state vector $X_{k}$ and control signal $U_{k}$ as follows:

$$
X_{k}=\left[\begin{array}{c}
x_{k} \\
x_{k-1} \\
\vdots \\
x_{k-p}
\end{array}\right] \in \mathbb{R}^{n(1+p)}, \quad U_{k}=\left[\begin{array}{c}
u_{k} \\
u_{k-1} \\
\vdots \\
u_{k-p}
\end{array}\right] \in \mathbb{R}^{n(1+p)} .
$$

The map $\varphi_{\text {est }}$ is defined as in (8) using the extended vector state. Observe that $x_{k}$ and $x_{k-p}$ are the first and last $n$ states of $X_{k}$, respectively:

$$
X_{k+p}=\varphi_{e s t}\left(k+p, k, X_{k}, U_{k}\left(X_{k}\right)\right) .
$$

The monodromy matrix for the periodic orbit of the extended system is obtained directly from (12). We define a new function (A.9), an extension of (11) for the DFC, and its Jacobian matrix for each point of the orbit is given by (A.10).

$$
\begin{aligned}
& \psi_{e s t}(X, K) \doteq\left[\begin{array}{cc}
0_{n \times n p} & 0_{n \times n} \\
I_{n p \times n p} & 0_{n p \times n}
\end{array}\right] X+\left[\begin{array}{c}
f\left(k,\left[I_{n \times n} 0_{n \times n p}\right] X\right)+K\left[-I_{n \times n} 0_{n \times n(p-1)} I_{n \times n}\right] X \\
------------------- \\
0_{n p \times 1}
\end{array}\right] \\
& \left.\nabla_{X} \psi_{e s t}(X, K)\right|_{X=X_{k}^{*}}=\left[\begin{array}{cc}
0_{n \times n p} & 0_{n \times n} \\
I_{n p \times n p} & 0_{n p \times n}
\end{array}\right]+\left[\begin{array}{c}
\left.\nabla_{x} f(k, x)\right|_{x=x_{k}^{*}}+K\left[-I_{n \times n} 0_{n \times n(p-1)} I_{n \times n}\right] \\
----------------- \\
0_{n p \times n(1+p)}
\end{array}\right]
\end{aligned}
$$




\section{References}

Alligood, K. T., Sauer, T. D. \& Yorke, J. A. [1996] Chaos an introduction to dynamical systems (SpringerVerlag, New York).

Bittanti, S. \& Colaneri, P. [2009] Periodic Systems: Filtering and Control (Springer Verlag, London).

Boukabou, A. \& Mansouri, N. [2007] "Fuzzy predictive controller for unknown discrete chaotic systems," International Journal of Bifurcation and Chaos 17, 2141-2148, doi:\{10.1142/S0218127407017318\}.

Chagas, T. P., Bliman, P.-A. \& Kienitz, K. H. [2010a] "Estabilização de órbitas periódicas: comparação entre realimentação de estados atrasados e uma nova lei utilizando estados preditos," XVIII Congresso Brasileiro de Automática (Bonito, Brazil).

Chagas, T. P., Bliman, P.-A. \& Kienitz, K. H. [2010b] "New feedback laws for stabilization of unstable periodic orbits," 8th IFAC Symposium on Nonlinear Control Systems (Bologna, Italy).

Chagas, T. P., Toledo, B. A., Rempel, E. L., Chian, A. C.-L. \& Valdivia, J. A. [2012] "Optimal feedback control of the forced van der pol system," Chaos, Solitons \& Fractals 45, 1147 - 1156, doi:10.1016/j. chaos.2012.06.004, URL http://www.sciencedirect.com/science/article/pii/S0960077912001282.

Chian, A. C.-L., Rempel, E. L. \& Rogers, C. [2006] "Complex economic dynamics: Chaotic saddle, crisis and intermittency," Chaos, Solitons and Fractals 29, 1194-1218.

Cvitanović, P. [1988] "Invariant measurement of strange sets in terms of cycles," Physical Review Letters 61, 2729-2732.

Franceschini, V., Giberti, C. \& Zheng, Z. [1993] "Characterization of the Lorentz attractor by unstable periodic orbits," Nonlinearity 6, 251-258.

Fung, Y. C. [2002] Theory of Aeroelasticity, Phoenix Edition Series (Dover Publications, Incorporated), ISBN 9780486495057.

Fussmann, G. F., Ellner, S. P., Shertzer, K. W. \& Hairston, N. G. [2000] "Crossing the Hopf bifurcation in a live predator-prey system," SCIENCE 290, 1358-1360, doi:\{10.1126/science.290.5495.1358\}.

Grasselli, O. M. \& Lampariello, F. [1981] "Dead-beat control of linear periodic discrete-time systems," International Journal of Control 33, 1091-1106, doi:10.1080/00207178108922978.

Hino, T., Yamamoto, S. \& Ushio, T. [2002] "Stabilization of unstable periodic orbits of chaotic discrete-time systems using prediction-based feedback control," International Journal of Bifurcation and Chaos 12, 439-446, doi:\{10.1142/S0218127402004450\}.

Huijberts, H., Michiels, W. \& Nijmeijer, H. [2009] "Stabilizability via Time-Delayed Feedback: An Eigenvalue Optimization Approach," SIAM Journal on Applied Dynamical Systems 8, 1-20, doi: $\{10.1137 / 070708767\}$.

Lagarias, J. C., Reeds, J. A., Wright, M. H. \& Wright, P. E. [1998] "Convergence Properties of the NelderMed Simplex Method in Low Dimensions," SIAM Journal of Optimization 9, 112-147.

Liz, E. \& Pötzsche, C. [2014] "PBC-based pulse stabilization of periodic orbits," Physica D 272, 26-38.

Mesquita, A., Rempel, E. L. \& Kienitz, K. [2008] "Bifurcation analysis of attitude control systems with switching-constrained actuators," Nonlinear Dynamics 51, 207-216, doi:10.1007/s11071-007-9204-7, URL http://dx.doi.org/10.1007/s11071-007-9204-7.

Morgül, Ö. [2009] "A new generalization of delayed feedback control," International Journal of Bifurcation and Chaos 19, 365-377.

Ott, E., Grebogi, C. \& Yorke, J. A. [1990] "Controlling chaos," Physical Review Letters 64, 1196-1199.

Parker, T. S. \& Chua, L. O. [1989] Practical Numerical Algorithms for Chaotic Systems (Springer-Verlag, New York).

Pyragas, K. [1992] "Continuous control of chaos by self-controlling feedback," Physics Letters A 170, 421428.

Sanjuán, M. A. F. \& Grebogi, C. [2010] Recent Progress in Controlling Chaos, Series on stability, vibration, and control of systems (World Scientific), ISBN 9789814291699.

Schmitt, D. T. \& Ivanov, P. C. [2007] "Fractal scale-invariant and nonlinear properties of cardiac dynamics remain stable with advanced age: a new mechanistic picture of cardiac control in healthy elderly," AMERICAN JOURNAL OF PHYSIOLOGY-REGULATORY INTEGRATIVE AND COMPARATIVE PHYSIOLOGY 293, R1923-R1937, doi:\{10.1152/ajpregu.00372.2007\}. 
Sontag, E. D. [1998] Mathematical Control Theory: Deterministic Finite Dimensional Systems, Texts in Applied Mathematics (Springer), ISBN 9780387984896.

Tian, Y. P. \& Zhu, J. D. [2004] "Full characterization on limitation of generalized delayed feedback control for discrete-time systems," Physica D 198, 248-257, doi:\{10.1016/j.physd.2004.09.005\}.

Turci, L. F. R., Macau, E. E. N. \& Yoneyama, T. [2009] "Efficient chaotic based satellite power supply subsystem," Chaos, Solitons $\&$ Fractals 42, 396 - 407, doi:http://dx.doi.org/10.1016/j.chaos.2008.12. 006, URL http://www.sciencedirect.com/science/article/pii/S0960077908005365.

Ushio, T. [1996] "Limitation of delayed feedback control in nonlinear discrete-time systems," IEEE Transactions on Circuits and Systems I - Regular Papers 43, 815-816.

Ushio, T. \& Yamamoto, S. [1999] "Prediction-based control of chaos," Physics Letters A 264, 30-35.

Yamamoto, S., Hino, T. \& Ushio, T. [2001] "Dynamic delayed feedback controllers for chaotic discretetime systems," IEEE Transactions on Circuits and Systems I - Regular Papers 48, 785-789, doi: $\{10.1109 / 81.928162\}$.

Yamamoto, S., Hino, T. \& Ushio, T. [2002] "Delayed feedback control with a minimal-order observer for stabilization of chaotic discrete-time systems," International Journal of Bifurcation and Chaos 12, 1047-1055, doi:\{10.1142/S0218127402004899\}, 1st Asia-Pacific Workshop on Chaos Control and Synchronization, SHANGHAI, PEOPLES R CHINA, JUN 28-29, 2001.

Zhu, H. D. \& Tian, Y. P. [2005] "Necessary and sufficient conditions for stabilizability of discrete-time systems via delayed feedback control," Physics Letters A 343, 95-107, doi:\{10.1016/j.physleta.2005. 06.007\}.

Zhu, J. \& Tian, Y.-P. [2008] "Stabilizability of uncontrollable systems via generalized delayed feedback control," Physica D 237, 2436-2443, doi:\{10.1016/j.physd.2008.03.029\}.

Zhuravlev, V. P. \& Klimov, D. M. [2010] "Theory of the shimmy phenomenon," Mechanics of Solids 45, 324-330, doi:10.3103/S0025654410030039, URL http://dx.doi.org/10.3103/S0025654410030039. 